\title{
Unsteady Aerodynamic Characteristics of Transversely Inclined Prisms Under Forced- Vibration-The Base Intensification Phenomenon
}

\section{Zengshun Chen}

Chongqing University

Jie Bai

Chongqing University

Cruz Y. Li ( $\nabla$ yliht@connect.ust.hk)

The Hong Kong University of Science and Technology https://orcid.org/0000-0002-9527-4674

Yemeng Xu

Chongqing University

Jianmin Hua

Chongqing University

Xuanyi Xue

Chongqing University

\section{Research Article}

Keywords: Forced-vibration technique, transversely inclined prisms, unsteady aerodynamics force, aerodynamic damping, response prediction

Posted Date: July 6th, 2021

DOI: https://doi.org/10.21203/rs.3.rs-609108/v1

License: (9) This work is licensed under a Creative Commons Attribution 4.0 International License.

Read Full License 
1 Unsteady Aerodynamic Characteristics of

Zengshun Chen ${ }^{\text {a }}$, Jie Bai ${ }^{\text {a }}$, Cruz Y. Li $^{\text {b,* }}$, Yemeng Xu ${ }^{\text {a }}$, Jianmin Hua ${ }^{\text {a }}$, Xuanyi Xue ${ }^{\text {a,* }}$

${ }^{a}$ School of Civil Engineering, Chongqing University, Chongqing 400045, China

${ }^{b}$ Department of Civil and Environmental Engineering, The Hong Kong University of Science and Technology, Clear Water Bay, Kowloon, Hong Kong, China.

zchenba@connect.ust.hk (Zengshun Chen); 202016131210t@cqu.edu.cn (Jie Bai);

yliht@connect.ust.hk (Cruz Y.Li); xu.ym@cqu.edu.cn (Yemeng Xu); hjm191@163.com (Jiamin Hua); xuexuanyi@126.com (Xue X. Y.)

E-mail address: yliht@ connect.ust.hk

Mailing address: School of Civil Engineering, Chongqing University, Chongqing 400045,

19 China

* Corresponding Author 
Abstract

This work, through a series of forced-vibration wind tunnel experiments, investigates the aerodynamic characteristics of square prisms subject to the transverse inclination. An aeroelastic prism was tested under different wind speeds, inclination angles, and oscillation amplitudes. Through analysis on the mean pressure distribution, local force coefficient, force spectra, and aerodynamic damping coefficient, the unsteady aerodynamic characteristics of the configuration were revealed. Empirical observations discovered the Base Intensification phenomenon, which refers to a fundamental change in the structure's aerodynamic behaviors given any degrees of transverse inclination. Specifically, it is the intensification of the aerodynamic loading, vortical activities, and aerodynamic damping on only the lower portion of an inclined structure. The phenomenon, being almost impactless to the upper portion, is also insensitive to changes in inclination angle and tip amplitude once triggered by the initial inclination. Analysis also revealed that the origin of Base Intensification phenomenon traces back to fix-end three-dimensional effects like the horseshoe vortex, instead of the predominant Bérnard-Kármán vortex shedding. Moreover, results showed that wind speed is the decisive factor for the structure's crosswind motions. Inside the lock-in region, structure loadings, vortical activities, and the effects of Base Intensification are significantly amplified. Beyond the range, the configuration gradually resorts to a quasi-steady linearity. Finally, results from the force-vibration tests were used for the prediction of structure response. Experimental comparison revealed that the predictions notably outperform those based on rigid tests, forecasting the actual responses with a markedly improved accuracy.

\section{Keywords}

Forced-vibration technique; transversely inclined prisms; unsteady aerodynamics force; aerodynamic damping; response prediction. 
Highlights:

$49 \checkmark$ A series of forced-vibration tests were performed to obtain unsteady aerodynamic forces

$50 \quad$ acting on a transversely inclined slender prism.

$51 \checkmark$ The nonlinear, unsteady aerodynamics characteristics of transverse inclination have been

$52 \quad$ analyzed.

$53 \quad \checkmark$ The Base Intensification unsteady phenomenon, which is unique to the transverse

54 inclination, has been identified and introduced.

$\checkmark$ The response of the prisms predicted by the unsteady aerodynamic forces; the newly proposed prediction method outperforms the traditional method based on rigid tests. 


\section{Introduction}

59 With the continuous improvement of construction technology, modern buildings are becoming

60 increasingly slender and flexible all over the world. Consequently, the influence of wind

61 loading and wind-induced vibration on super-tall buildings are of great safety concern,

62 particularly after being remined by the recent vibration of the SEG Tower in Shenzhen.

63 Therefore, studies on the aerodynamic characteristics of super-tall buildings bear critical

64 importance. To date, much remain unexplored for the even the most canonical configurations,

65 for example the square prism. To tackle the outstanding issues, the deepening of our understanding of bluff-body aerodynamics demands persistent effort.

The aerodynamic characteristic of a structure is the key determinant to its wind-induced response. In the event of excessive excitations, whether by the Vortex-Induced-Vibration (VIV), galloping, or fluttering, irreversible damage may be incurred on the structure. In many cases, the modes of vibration are closely associated to the aerodynamic damping, because a negative aerodynamic damping will aggravate the vibration until plastic deformation. Therefore, a series of experimental research have been performed on the aerodynamic characteristics of bluff-bodies. Tanaka et al. [1] studied the aerodynamic characteristics of unconventional building models (spiral, cone, etc.) through rigid pressure test. Hui et al. [2] examined the influence of different façade appendages on the aerodynamic characteristics of high-rise buildings. Likewise, Carassale et al. [3] studied the influence of round corners on the aerodynamic characteristics of rectangular prisms. However, these studies are limited to the 
scenarios when the structure is aligned vertically perpendicular to the incoming flow, which might not be the case for many modern designs of infrastructure. To this end, Hu et al. [4] studied the longitudinally inclined (forward and backward) rectangular prism through a series of wind tunnel tests. They concluded that the inclination angle has profound impacts on the aerodynamic characteristics of prisms, yet the degrees of the impact are significantly different depending on the inclination angle. Perhaps the biggest limitation to this work is the rigidity of the test model. Due to the complex behaviors of the fluid and its mutually reinforcing interactive mechanisms with the structure, it is difficult to evaluate the unsteady aerodynamic forces accurately using a rigid model. Aeroelasticity must be included to accommodate the fluid-structure interaction. Subsequently, Hu et al. [5] confirmed the quasi-static theory inadequate for predicting inclined structural responses using an aeroelastic model. Mannini et al. [6] too disproved the theory for low mass-damping structures. Later, Chen et al. [7] discovered the Partial Reattachment phenomenon for aeroelastic tapered prisms, in which the closure of a separation envelope to a separation bubble by increasing the wind attack angle suppresses the Bérnard-Kármán vortex shedding altogether. Indeed, the quasi-static theory also failed in the prediction of tapered prisms. Research showed that structures with shape irregularities generally disobey the quasistatic theory. The fundamental cause is the neglect of the unsteady effects, which is only captured by aeroelastic measurement. In other word, ignoring the unsteady effects brought about by aeroelasticity is equivalent to a quasi-static prediction, which is bound to deviate from reality. 
102 To this end, previous research proved that the forced-vibration method produce relatively 103 accurate evaluations of the unsteady effects [8-10]. Kim et al. [11] examined the impact of 104 vibration amplitude on the aerodynamic forcing of a structure, finding notable improvements by forced-vibration compared to the rigid test. Furthermore, Chen et al. [12, 13] systematically compared the aerodynamic forces measured from rigid, forced-vibration and the hybrid aeroelastic-pressure balance (HAPB) test (i.e., free-vibration). Their analysis showed that the HAPB, being the optimal testing method among the three, accurately depicts the bi-directional feedback loop of the fluid-structure coupling, whereas this loop is utterly absent for the rigid test and unidirectional for the forced-vibration test.

In addition, Chen et al. [14] studied the unsteady aerodynamic forces acting on a rectangular prism using the forced-vibration method, finding that the permission of vibration significantly

114 alters the aerodynamic force coefficient in the crosswind direction, while only minimally

115 affecting those in the alongwind direction. Interestingly, they also found the generalized and local root-mean-square (RMS) lift increases with oscillation amplitude only at low wind speed and remains relatively constant at high wind speed. Furthermore, based on [4] and a series of aeroelastic wind tunnel tests, it was shown that both the oscillation amplitude and wind speed are the key factors affecting the aerodynamic damping of longitudinally inclined prisms, especially in the lock-in region [9]. The inclination angle per se may also lead to major deviations of the aerodynamic damping from the vertical case. 
123 The thorough literature review discloses a critical void in bluff-body aerodynamics, that is, 124 there is yet a study on the transversely inclined prism with the aeroelastic effect. Yet the 125 configuration is highly probable given variations of the wind attack angle. Intuitively, the transverse inclination will modify the aerodynamic characteristics of structures and their surrounding flow field. Perhaps the only referential effort was the one that proposed a modified quasi-steady model for transversely inclined prisms [15]. However, the predicted response was only acceptable for the onset of galloping but notably off thereafter. Therefore, the aerodynamic characteristics brough about by transverse inclination, and the feasibility of the quasi-steady theory for this configuration demand further investigation.

This work aims to examine the aerodynamic characteristics of transversely inclined prisms.

The unsteady aerodynamic forces acting on the model were obtained through a series of forcedvibration tests, and the aerodynamic characteristics of the model were analyzed under representative wind speeds, inclination angles, and vibration amplitude. In composition, Section 1 offers the contextual information and a thorough literature review on the topic of investigation. Section 2 details the methodology of the forced-vibration wind tunnel test. Section 3 presents the identification of the model's aerodynamic characteristics from test results, and elucidates the influence of inclination angle on the aerodynamic characteristics. 
142 damping, and the comparative observations with the aeroelastic results. Section 5 summarizes

143 the major findings of this work.

144 


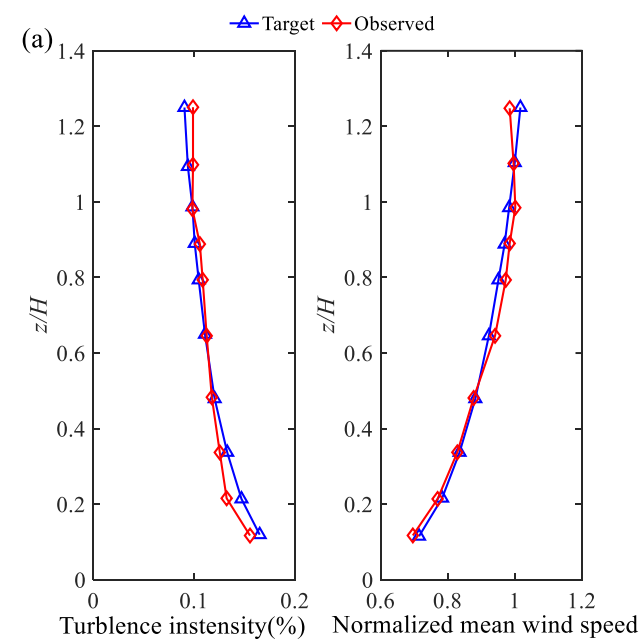

(a) turbulence intensity and wind profile

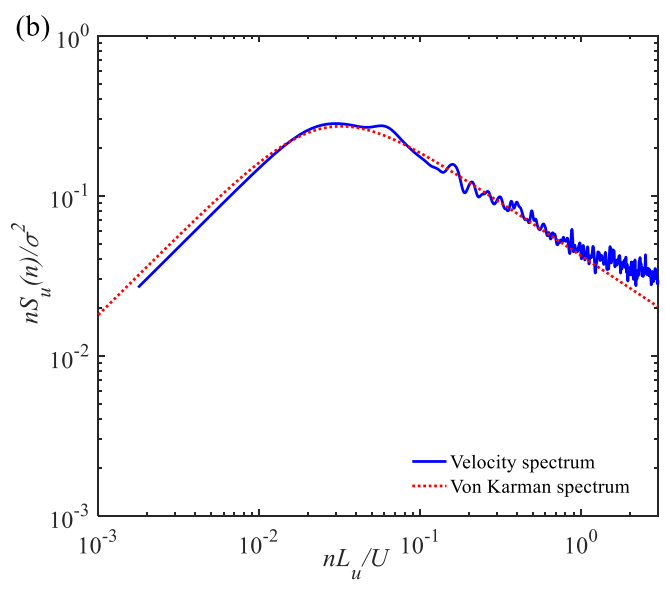

(b) the wind speed spectrum

Fig. 1 Wind field characteristics 


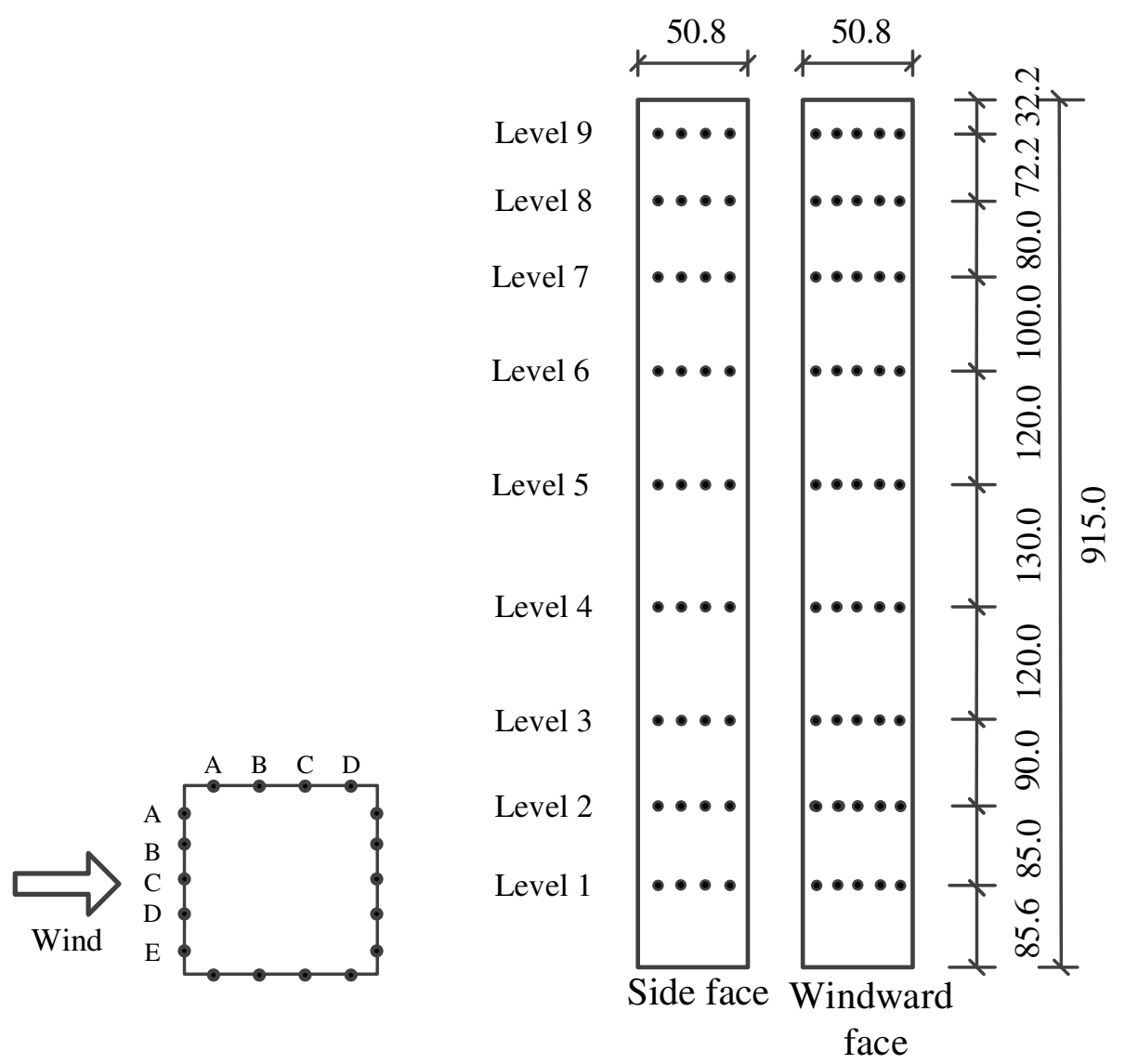

(a)

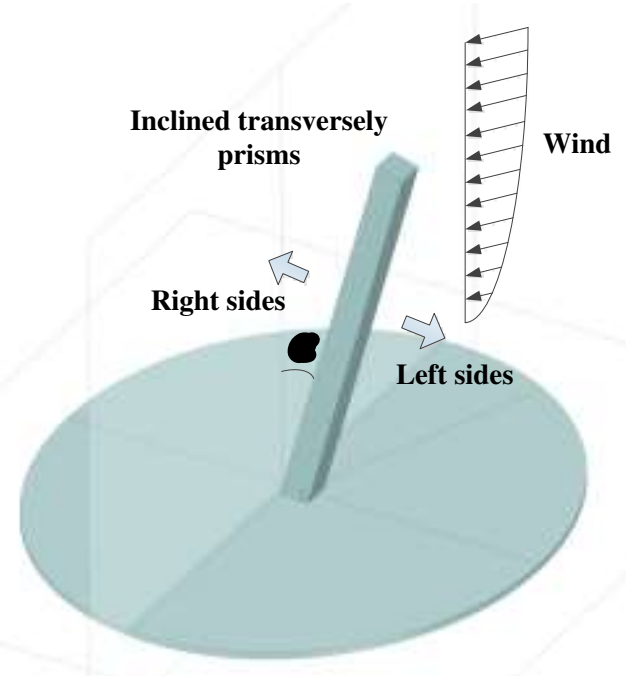

(b) 

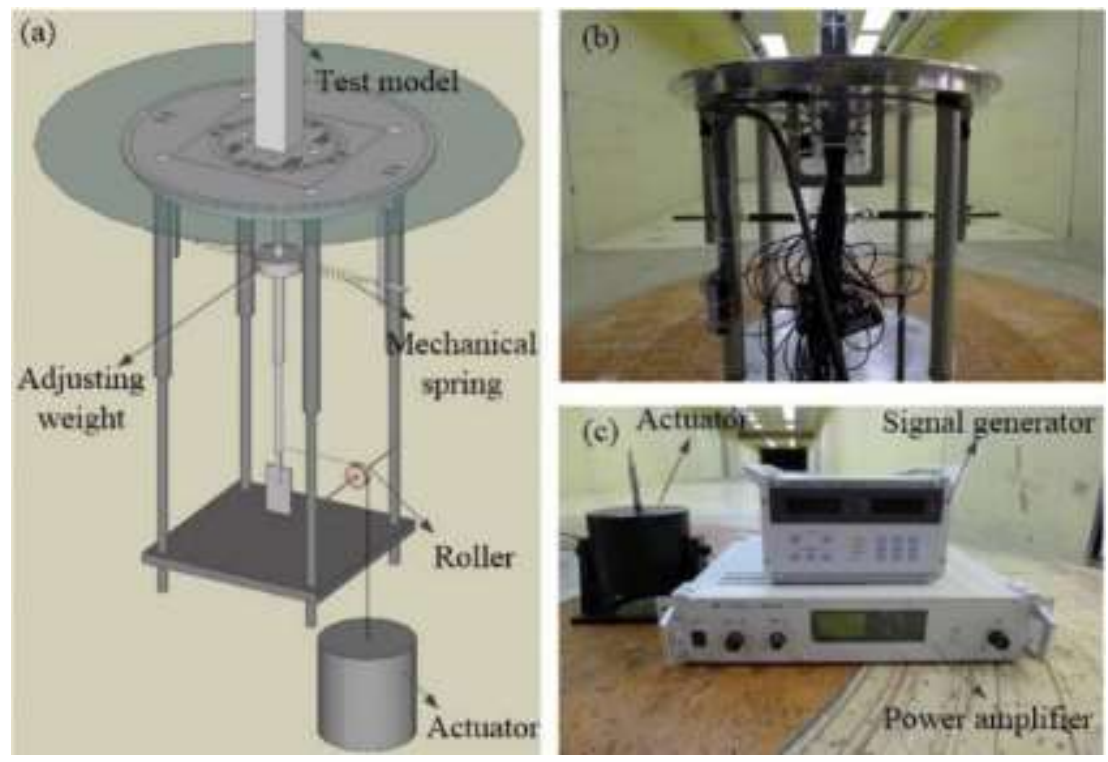

(c)

Fig. 2 Model for pressure measurements: (a) The distribution of Pressure taps; (b) transversely inclined prisms; (c) The forced-vibration device and system

146 The main investigative method of this work is wind tunnel experiment, which proved one of 147 the most effective method for endeavors in bluff-body aerodynamics. The wind tunnel tests 148 were performed in the high-speed section of the CLP Power Wind/Wave Tunnel Facility at the 149 Hong Kong University of Science and Technology. The dimension of the wind tunnel was 29.2 $\mathrm{m}$ (Length) $\times 3 \mathrm{~m}($ Width $) \times 2 \mathrm{~m}$ (Height). The corresponding the blockage ratio was $0.78 \%$,

151 with which undesired influence of tunnel walls on the wind field can be avoided [16]. The mean wind profile exponent $\beta$ was determined as 0.15 , and the turbulence intensity at the model top was $10 \%$. The corresponding provisions in the specification were compared with the wind characteristics measured in this test, as shown in Fig. 1

(a) and (b). The comparison shows that the simulated wind characteristics closely replicate the target ones, therefore are appropriate for wind tunnel testing. 
Table 1 Basic parameters of the model and wind tunnel test

\begin{tabular}{|c|c|c|c|c|c|c|}
\hline $\begin{array}{l}\text { Height } \\
(\mathrm{mm})\end{array}$ & $\begin{array}{l}\text { Pressure } \\
\text { Taps }\end{array}$ & $\beta$ & $\begin{array}{l}\text { Prism } \\
\text { Length } \\
(\mathrm{mm})\end{array}$ & $\begin{array}{l}\text { Reduced Wind } \\
\text { speeds }\end{array}$ & $\begin{array}{c}\text { Oscillation } \\
\text { Amplitude }(\%)\end{array}$ & $\begin{array}{l}\text { Inclination } \\
\text { Angle } \alpha\left(^{\circ}\right)\end{array}$ \\
\hline 915 & 162 & 0.15 & 50.8 & $\begin{array}{c}6,7,8,8.5,9,9.5 \\
10,10.5,11,11.5 \\
12,13,14,15,16 \\
18,20\end{array}$ & $\begin{array}{c}0,8,10,12, \\
14,16,18,20\end{array}$ & $\begin{array}{c}0,5,10,15 \\
20,30\end{array}$ \\
\hline
\end{tabular}

The test model was a square prism with the dimension $50.8 \mathrm{~mm}(B) \times 50.8 \mathrm{~mm}(D) \times 915 \mathrm{~mm}(H)$, where $B$ and $D$ were the length and width of the cross-section and $H$ was the height (Fig. 2 ).

The aspect ratio of the model was 18:1. The natural vibration frequency of the model was set to $7.8 \mathrm{~Hz}$. In the forced-vibration test, a device installed under the base of the model initiated harmonic excitations. The inclination angle $\alpha$ of the model varied from $0^{\circ}$ to $30^{\circ}$, and the amplitudes of oscillation was prescribed to be between $6 \%$ and $20 \%$.

Reduce wind speed $V_{r}=U / f D_{c}$ ( $U$ is the wind speed at the top of the model, $f$ is the natural frequency, and $D_{c}$ is the characteristic length) was determined to be between 6 and 20. More experimental details are summarized in Table 1 . The sampling frequency and the duration were set to $500 \mathrm{~Hz}$ and $100 \mathrm{~s}$. The tip response of the model was obtained by correcting the raw data measured from the strain gauge at the model base. The unsteady wind pressure on the model was measured by a Multi-Point Synchronous Pressure Measurement System (SMPSS). Fig. 2 (a) shows the distribution of Pressure Taps. It should be emphasized that the pressure and the response of the test model were measured synchronously. To avoid literary redundancy, more

174 details can be found in [9], which adopted the same testing parameters. 

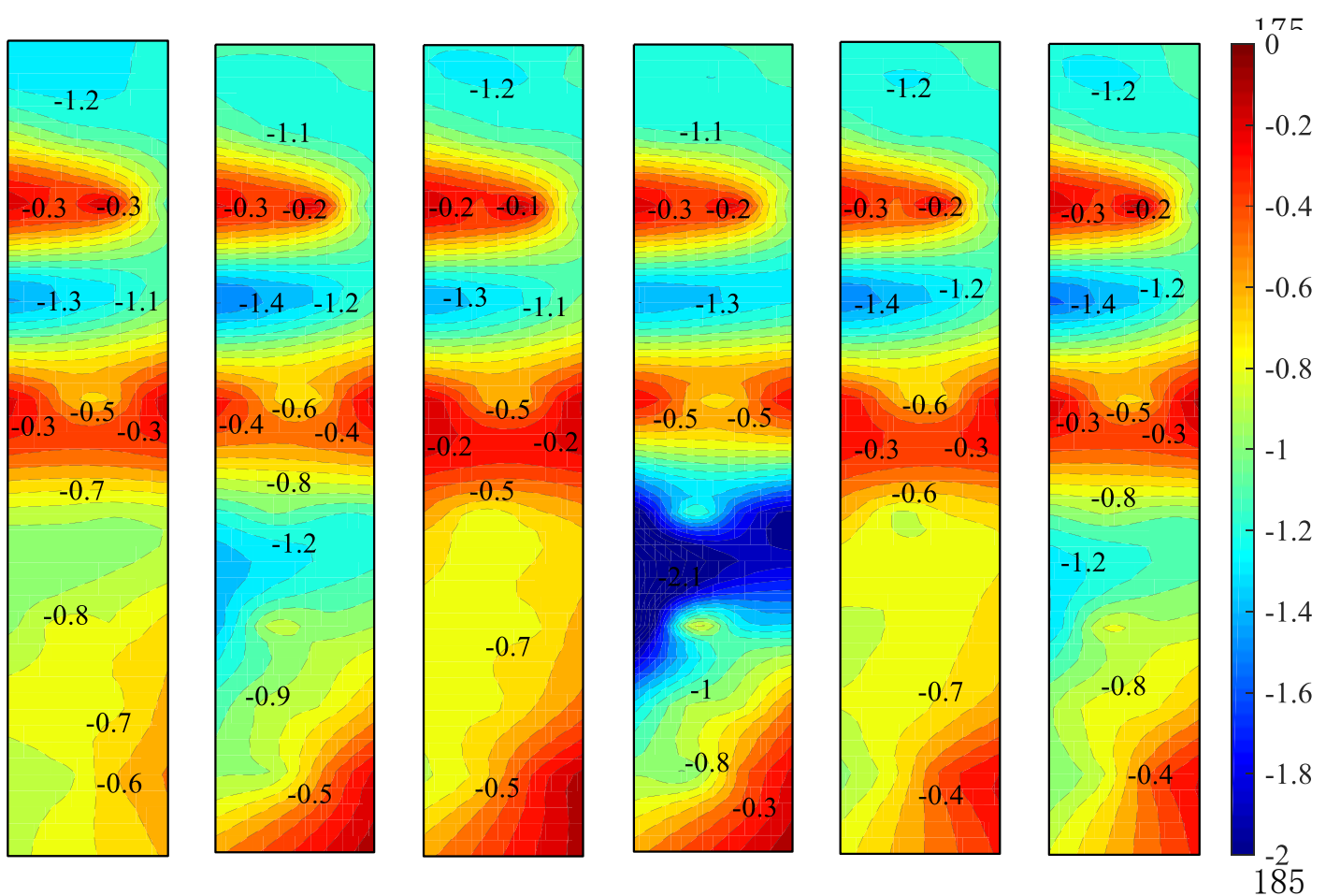

$\alpha=0^{\circ}$

$$
\alpha=5^{\circ}
$$
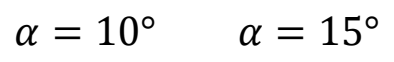

$$
\alpha=20^{\circ}
$$$$
\alpha=30^{\circ}
$$

186

Fig. 3 Mean pressure distribution on the left $\operatorname{side}\left(V_{r}=11\right)$
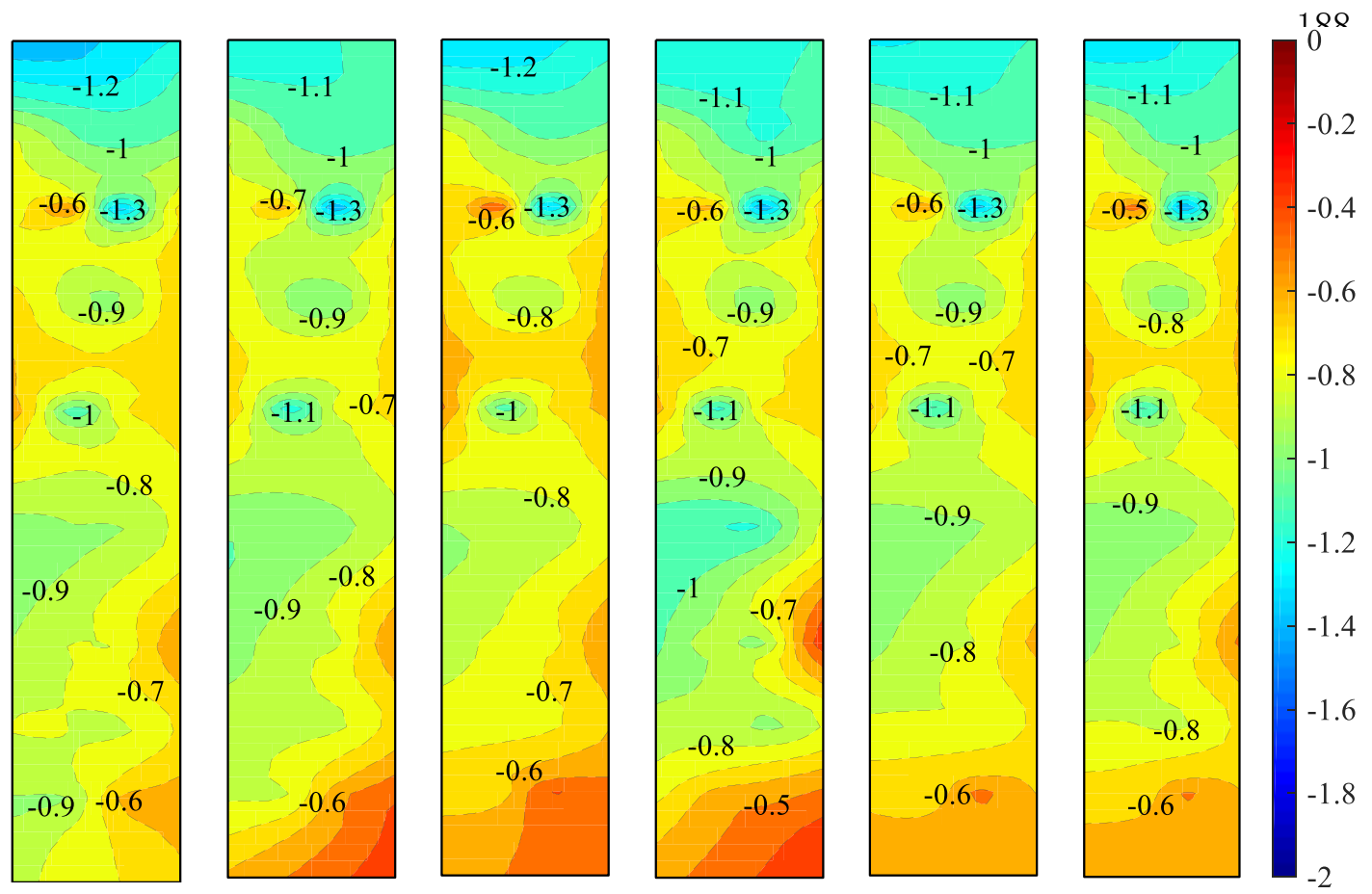

$$
\alpha=0^{\circ}
$$

$$
\alpha=5^{\circ}
$$$$
\alpha=10^{\circ}
$$

$\alpha=15^{\circ}$

$\alpha=20^{\circ}$

$\alpha=30^{\circ}$

Fig. 4 Mean pressure distribution on the right side $\left(V_{r}=11\right)$ 

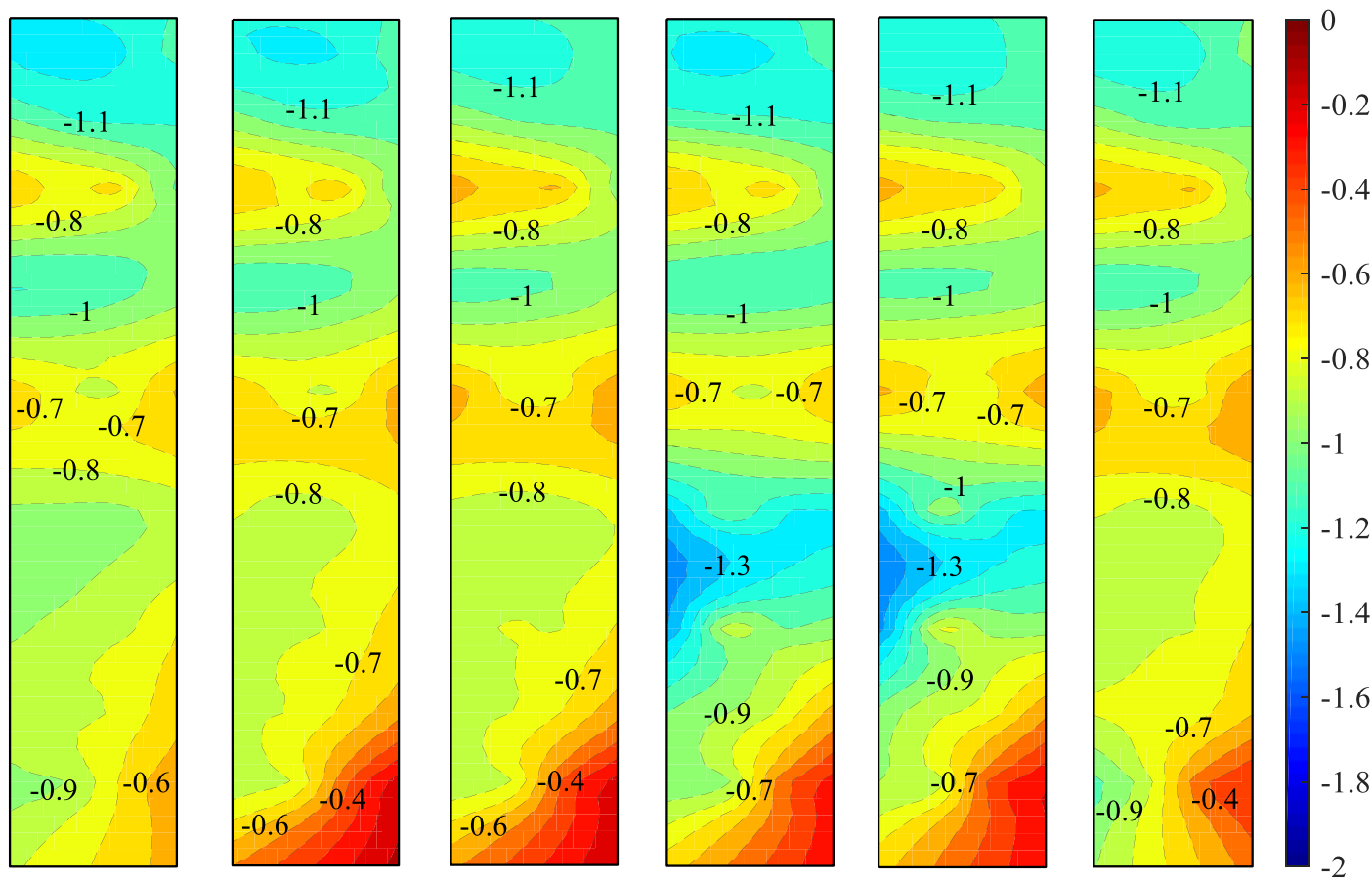

$\alpha=0^{\circ}$

$$
\alpha=5^{\circ}
$$$$
\alpha=10^{\circ}
$$

$\alpha=15^{\circ}$

$\alpha=20^{\circ}$

$\alpha=30^{\circ}$

Fig. 5 Mean pressure distribution on the left side $\left(V_{r}=18\right)$
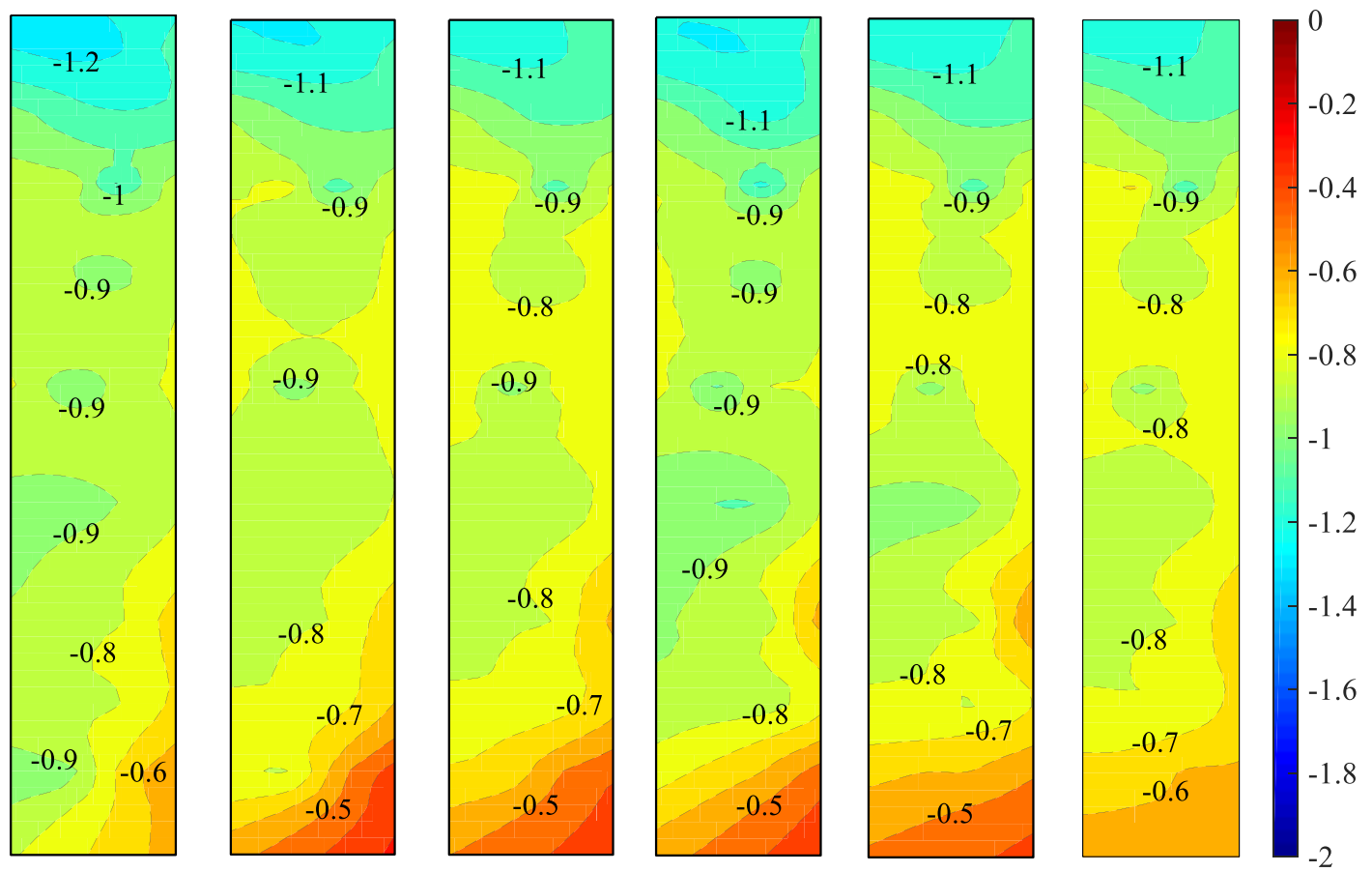

$\alpha=0^{\circ}$

$$
\alpha=5^{\circ}
$$$$
\alpha=10^{\circ}
$$

$\alpha=15^{\circ}$

$\alpha=20^{\circ}$

$\alpha=30^{\circ}$

Fig. 6 Mean pressure distribution on the right $\operatorname{side}\left(V_{r}=18\right)$ 

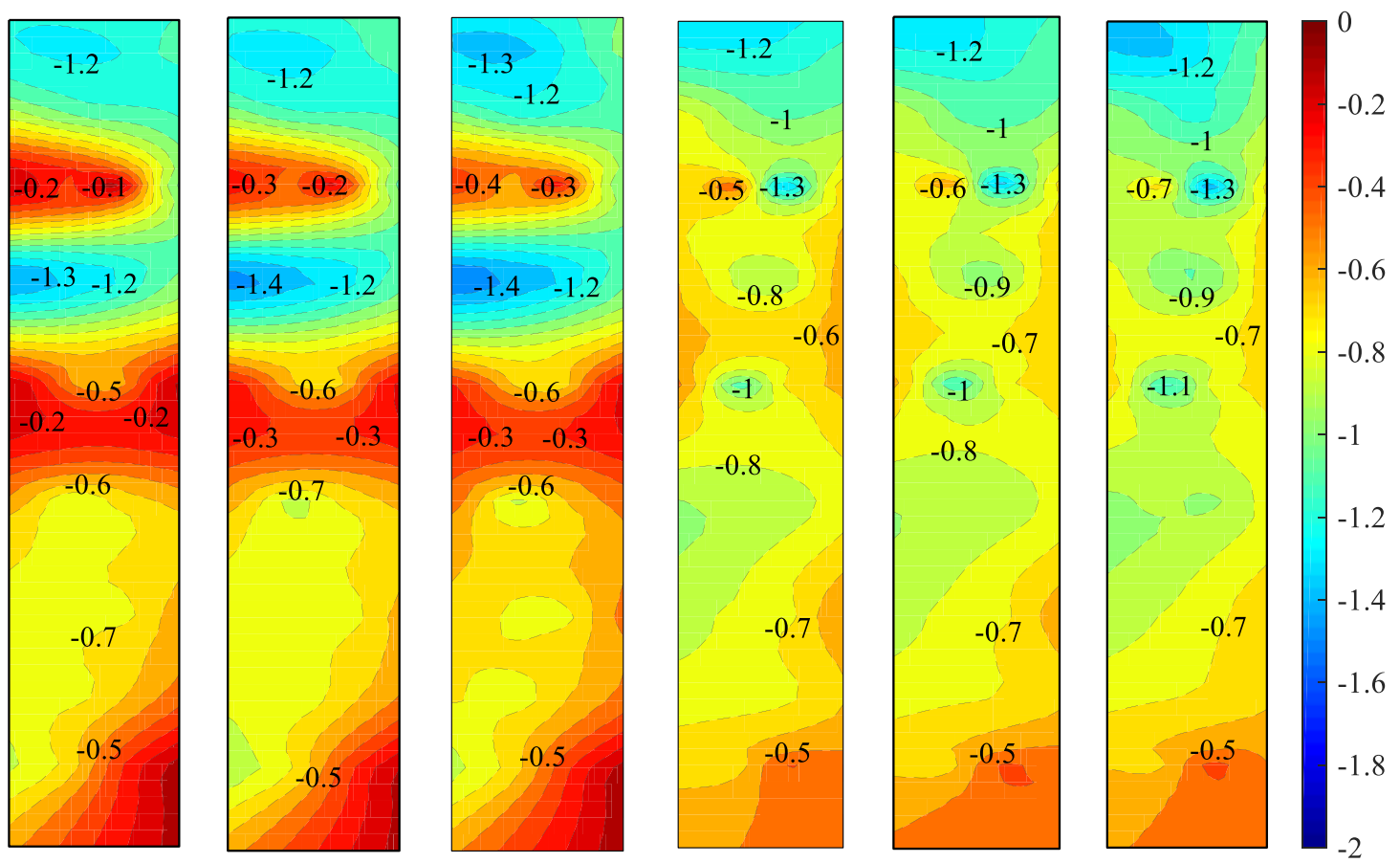

$\sigma_{y} / D=0 \%$

$\sigma_{y} / D=8 \%$

$\sigma_{y} / D=18 \%$

$\sigma_{y} / D=0 \%$

$\sigma_{y} / D=8 \%$

$\sigma_{y} / D=18 \%$

Left face

Right face

Fig. 7 Mean pressure distribution on both sides $\left(V_{r}=11 \alpha=10^{\circ}\right)$
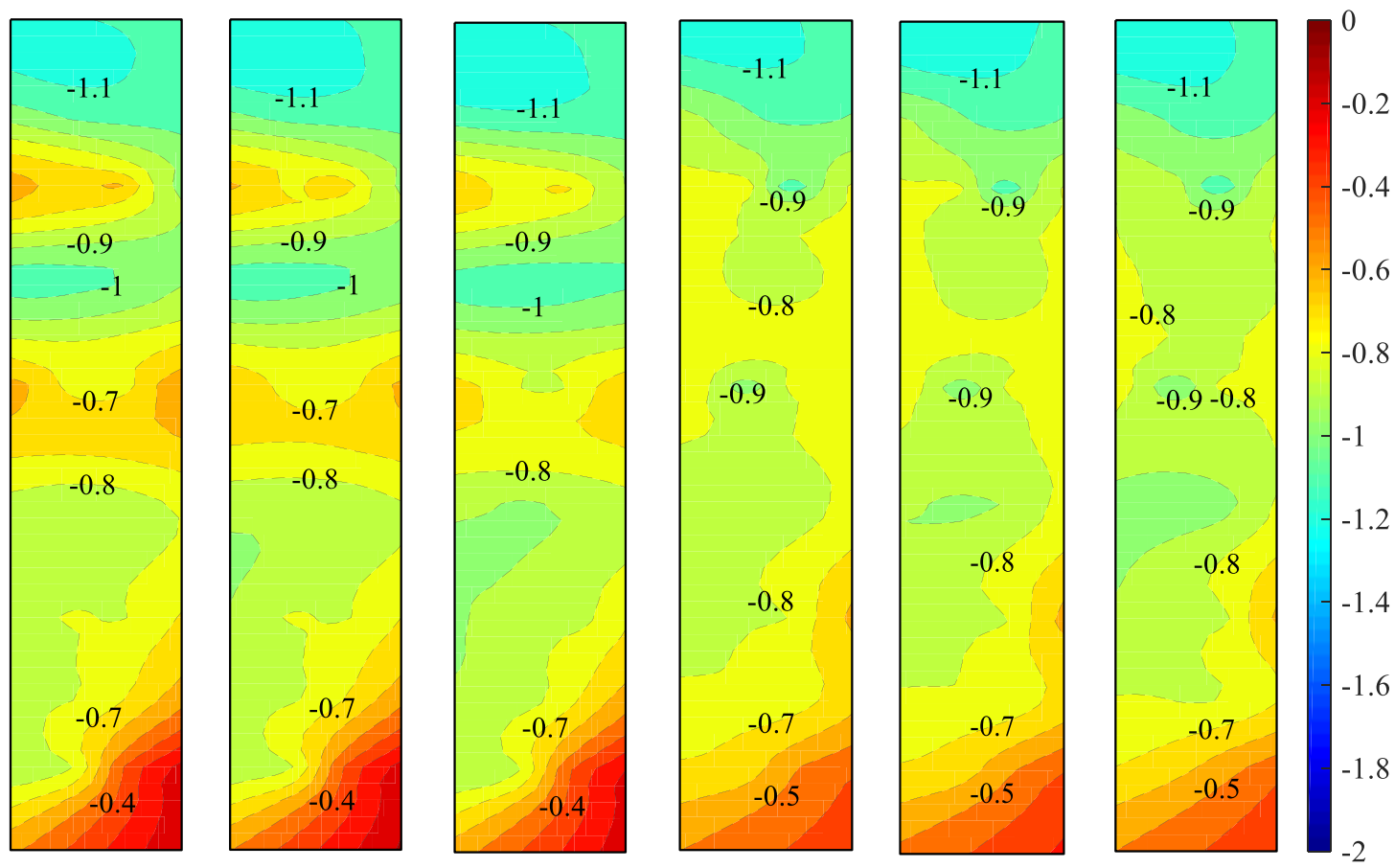

$\sigma_{y} / D=0 \%$

$\sigma_{y} / D=8 \%$

$\sigma_{y} / D=18 \%$

$\sigma_{y} / D=0 \%$

$\sigma_{y} / D=8 \% \quad \sigma_{y} / D=18 \%$

Left face

Right face 
Fig. 8 Mean pressure distribution on both sides $\left(V_{r}=18 \alpha=10^{\circ}\right)$

202

203

204

205

206

207

208

209

210

211

212

213

214

215

216

217

218

219

220

\section{Results and Discussion}

\subsection{Unsteady Aerodynamic Coefficient}

\subsubsection{Mean Pressure Distribution}

Equation (1) was used to calculate the mean wind pressure coefficient $C_{P}$ acting on the model:

$$
C_{P}=\frac{2 P_{i}}{\rho U^{2}}
$$

where $P_{i}$ is the local pressure acting on the model and $\rho$ is the density of air.

Fig. 3-Fig. 8 present the mean pressure distribution of the model surface at different inclination angles $\alpha$ and vibration amplitude $\sigma_{y} / D$. Due to the change of wind attack angle as the result of transverse inclination, the flow separation at the leading edge is not exactly symmetrical. Therefore, perhaps the most representative observations come from pressure distributions on the side faces. Given the abundance of test results, the most insightful sets of data, with wind speeds of $V_{r}=11$ and $V_{r}=18$ and vibration amplitudes $\sigma_{y} / D=0 \%, 8 \%$, and $18 \%$, are presented. $V_{r}=11$ is characteristic of the lock-in region and $V_{r}=18$ is characteristic of the high wind speed region. It is also important to define the orientation of the side faces for the convenience of subsequent discussion. As shown in Fig. 2 (b), the left side refers to the nearwind side that faces the ground. The right side refers to the far-wind side that faces the ceiling.

In the most general sense, the side faces of the model experiences suction (negative pressure).

The sharpness of pressure gradients seems to be inversely proportional to the wind speed, 
221 although this might be directly attributed to the lock-in effect. In addition, the pressure distribution on either faces are completely different, where dynamical activities on the left face are of greater intensity and subject to more prominent changes with $\alpha$. Moreover, the lower half of the model is greatly affected by $\alpha$, whereas the upper half remains relatively insensitive.

Specifically, we examine the side faces in parallel for better comparisons. Fig. 3 and Fig. 4 show the wind pressure distribution of the left and right faces of different $\alpha$ at velocity $V_{r}=$ 11, and no forced-vibration $\sigma_{y} / D=0 \%$. On the right side, $\alpha$ has limited influence on the pressure of the top half of the model. However, the model base is quite sensitive to $\alpha$ : low suction zones propagate from the rear corner of the model base towards higher level, before being thwarted near the mid-span. In this lock-in region, $\alpha$ promotes the restoration of pressure most prominently at $15^{\circ}$. In addition, the maximum pressure on the right side is $C_{P}=-1.3$ at the Level 8, which stays fairly consistent at all $\alpha$ 's.

In comparison to that of the right side, the pressure distribution on the left side is more sophisticated. First, the top half of the model remains unaffected by $\alpha$, but a region of sharp pressure restoration $\left(C_{P}=-0.2\right.$ and -0.3$)$ persists at about $1 / 5 H$ from the free-end. The location corresponds precisely with that of the maximum pressure on the right side. At the model mid-span, a sharp band of high-pressure zone runs across the entire model width, which is consistently maintained with only slight alterations at $\alpha=10^{\circ}$ and $15^{\circ}$. Conversely, the 
242 the rear end of the base is evident. The restoration is accompanied by a sharply propagating 243 pressure gradient. Yet, perhaps the most drastic and unexpected change occurs at $\alpha=15^{\circ}$. A 244 region of extreme suction, at $C_{P}=-2.1$, occurs just below the mid-span high-pressure band.

245 Just below this suction zone, another sharp pressure gradient takes place and restores pressure 246 back to as high as $C_{P}=-0.3$. From an aerodynamics point-of-view, the logical explanation 247 is that at $\alpha=15^{\circ}$, an intense separation bubble forms, which is associated to reattachment and 248 the impingement of certain types of vortices. However, the affiliation of these vortices requires 249 further evidence. With the increase of wind speed (Fig. 5 and Fig. 6) beyond the lock-in region to $V_{r}=18$, the most visible change is the moderation of extremities in the pressure distribution on either side.

253 Evidently, the pressure patterns for the right side resembles those of the lock-in case, except 254 displaying less variability and milder extreme values. For example, the observed maxima reduced from -1.3 to -1.0 . On the left side, similar observations are made. Features like the leading-edge restoration near the free-end, mid-span restoration band, and the suction zone all appear with much milder intensity. Interestingly, near the model base, the pattern and intensity of the pressure distribution are almost identical between $V_{r}=11$ and 18. This is to say, knowing the lock-in is excited by the resonance between the Karman vortex and structure motion, the vortical activities on the model base are not associated with the Bérnard-Kármán vortex shedding. Instead, they are perhaps associated with the horse-shoe vortex that occurs universally near the base of three-dimensional bluff-bodies. 
264 Next, to elucidate the influence of vibration amplitude on wind pressure distribution, Fig. 7 265 and Fig. 8 present a comparison of the mean wind pressure distribution at $\alpha=10^{\circ}$, wind speed 266 at $V_{r}=11$ and $V_{r}=18$, and amplitude at $0 \%, 8 \%$ and $18 \%$. Clearly, pressure distribution is 267 only almost insensitive to the vibration amplitude. Although minute variations can be observed, 268 but when compared to the degree of change in vibration amplitude (i.e., from 0-18\%), such differences are trivial.

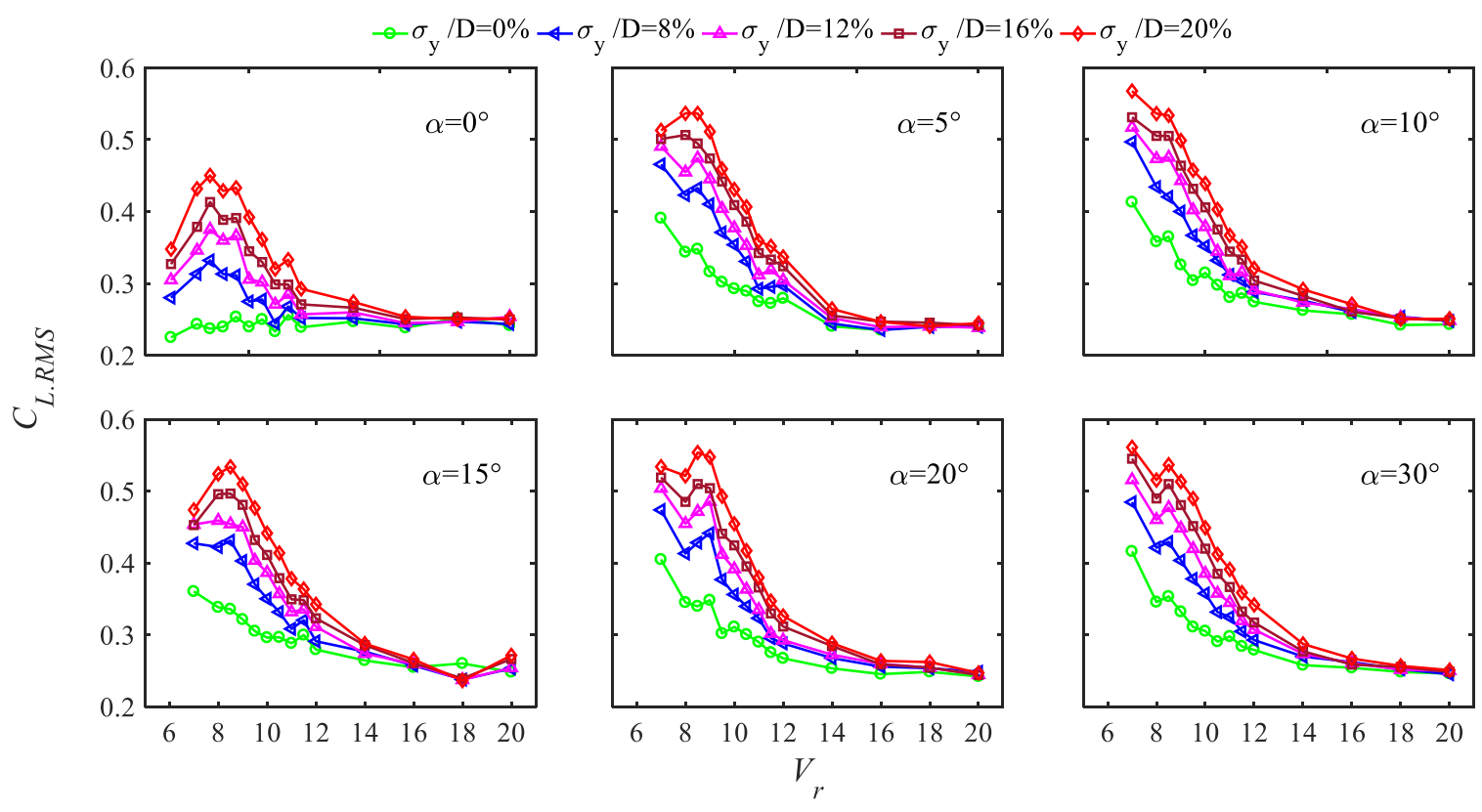

Fig. 9 Generalized RMS force coefficients

272 The unsteady aerodynamic force coefficient in the crosswind direction can be obtained as: 


$$
\begin{gathered}
\tilde{C}_{L, r m s}(z)=\frac{2 \tilde{F}_{L}(z)}{\rho A(z) U^{2}} \\
\tilde{C}_{L, r m s}=\frac{\int_{0}^{H} A(z) \tilde{C}_{L}(z) \phi(z) d z}{D H}
\end{gathered}
$$

273 where $\tilde{C}_{L, r m s}(z)$ is local RMS force coefficient and $\tilde{F}_{L}(z)$ is RMS lift force. Equation (3) is 274 obtained by integrating Equation (2) along the model height, in which $A(z)$ is the local area at height $z, \phi(z)$ is the function of the first mode, namely $\phi(z)=z / H$.

Since the mean lift coefficient is approximately zero, only the RMS lift force coefficient bears significant implications [17]. Fig. 9 presents the variation of the generalized RMS lift coefficient at different $\alpha$, wind speeds and vibration amplitudes. The significant influences of $\alpha$ and vibration amplitude on the RMS lift are limited to the low-speed range, as all variations collapse into a single curve around 0.25 after $V_{r}=16$. This observation supports the quasisteady notion at sufficiently high wind speeds. On the other hand, the proportionality between the RMS lift and vibration amplitude is uniform for all $\alpha$, where the self-similar curves are simply shifted vertically in the low-speed range. Reminded by the observations on the mean pressure, from which no obvious dependence on vibration amplitude could be deduced, it is concluded that the primary effect of the vibration amplitude is projected onto the RMS force. This is to say, increasing the vibration amplitude enhances the turbulence activities of the surrounding field, so that the intensified fluctuating wind field transfers the added turbulent 
289 kinetic energy (TKE) onto the structure as fluctuating pressure. The extra TKE is attributed to 290 the added kinetic energy from the enlarged vibration amplitude and reflects this intensification 291 of the unsteady effects.

293 With a rather straightforward proportionality of the vibration amplitude, the effect of $\alpha$ on the 294 RMS lift becomes lucid. First, an increase of $\alpha$, regardless of the extent, significantly amplifies 295 the RMS lift by at least $20 \%$ (some even to 100\%). Second, compared to the vertical case $(\alpha=$ $\left.2960^{\circ}\right)$, any alteration of $\alpha$ induces the lock-in effect for the non-vibrating case $\left(\sigma_{y} / D=0 \%\right)$. 297 Finally, an increase of $\alpha$ only minimally affect the maximum RMS lift, as its magnitude 298 generally floats around 0.55 . All these observations point to a single fact: the aerodynamic 299 characteristics of the configuration experience a sudden change after prescribing an $\alpha$ to the 300 vertical case, but once taken place, further increase of $\alpha$ project minimal impacts. 

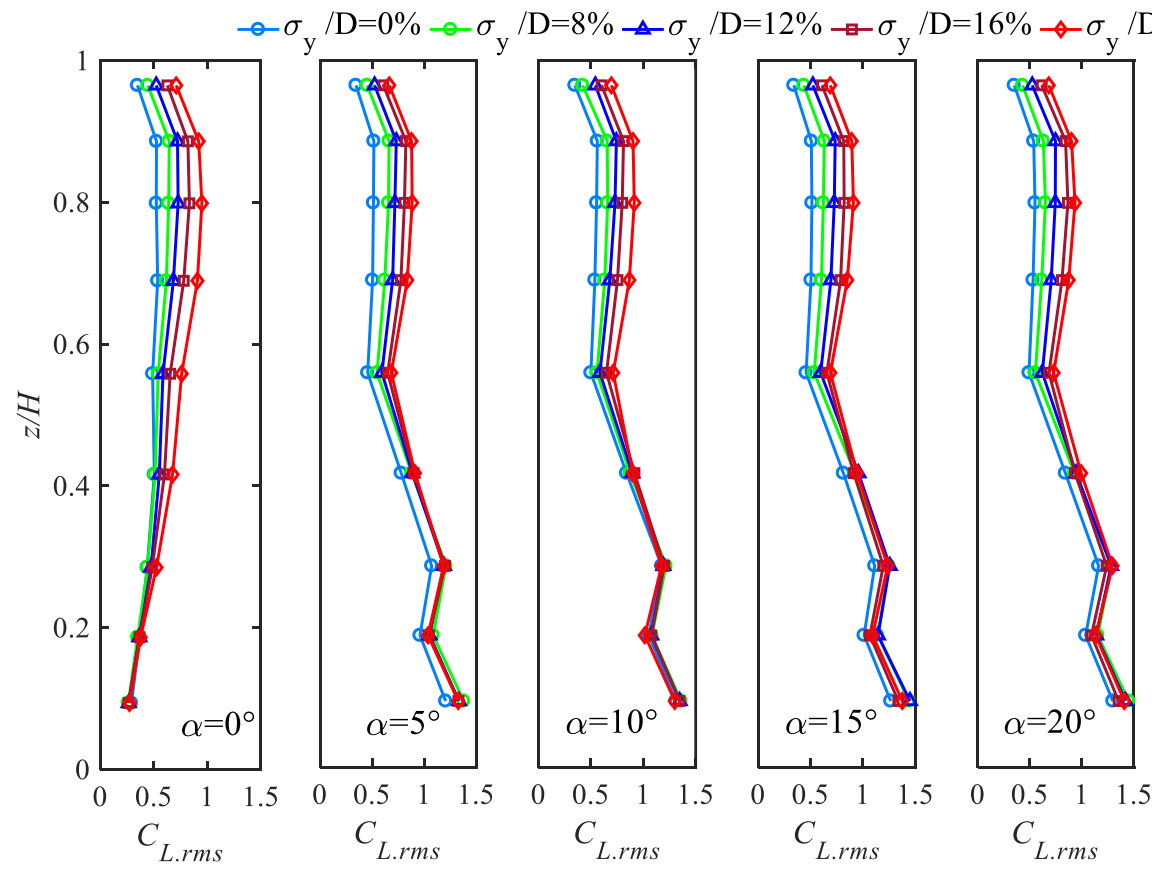

$\mathrm{D}=20 \%$

Fig. 10 Local RMS lift coefficient in the low wind speed $\left(V_{r}=11\right)$
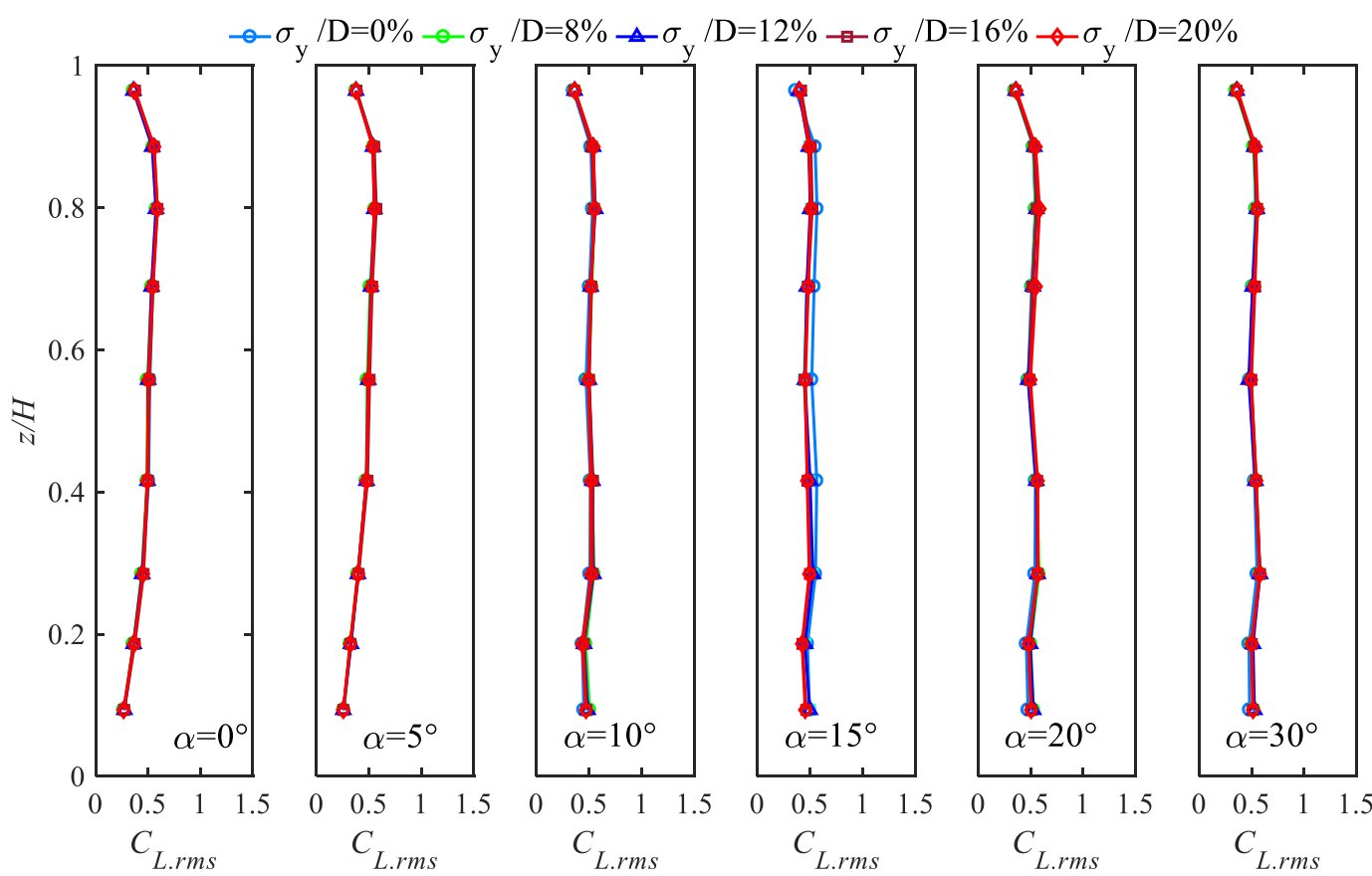

Fig. 11 Local RMS lift coefficient in the high wind speed $\left(V_{r}=18\right)$

To instigate better understanding of the interaction between the structure and fluid, the local

RMS lift force coefficients showing the distribution of aerodynamic forces along the model 
height is calculated by Equation (2). Fig. 10 and Fig. 11 present the local RMS lift in the lockin region $\left(V_{r}=11\right)$ and in the high wind speed $\left(V_{r}=18\right)$ at vibration amplitudes $0 \%, 8 \%, 12 \%$, $16 \%$, and $20 \%$.

For the lock-in case (Fig. 10), the bi-polar change induced by $\alpha$ is clear. The vertical case differs fundamentally from all other cases. The local RMS lift of the vertical case maximizes at Level $8(z / H=0.8)$, whereas those of all other cases maximize at the model base $(z / H=0.1)$. In fact, the profiles of all the cases with altered $\alpha$ are self-similar, showing that, after the initial perturbation, a further increase of $\alpha$ does not affect the unsteady forces acting on the model. Furthermore, by close inspection, the profiles can be divided into two halves separated at Level 5. A prescription of $\alpha$ only promotes the RMS lift on the bottom half, the lower the more significant. Yet, $\alpha$ minimally, if at all, affects the upper half of the model. This observation echoes with the mean field distribution in Fig. 3 and Fig. 4. By contrast, the vibration amplitude affects only the upper half of the model, as an increase of amplitude instigates a proportional increase in the RMS lift.

The high-speed case (Fig. 11) displays shows the local RMS is generally uniform across the model height and unaffected by either $\alpha$ or vibration amplitude. The collapse of all self-similar curves into a single curve reinforces the quasi-steady notion for this range of wind speeds. 
maximum RMS lift for the upper half does not occur at the free-end but immediately below.

This observation bears critical importance and will be discussed in the subsequent sections.

\subsection{Power Spectral Density}

\subsubsection{Generalized Force Spectra}

Fig. 12 and Fig. 13 present the generalized force spectra in the crosswind direction at $\alpha=0^{\circ}$, $15^{\circ}$ and $30^{\circ}$. The $\sigma_{y} / D=0 \%$ case represents model with infinite rigidity, which does not consider any fluid-structure interaction. As the result, only one peak [18] corresponding to the frequency of the Bérnard-Kármán vortex shedding is observed. With vibration amplitudes, the forced-vibration tests consider the one-way fluid-structure interaction (i.e., from structure to fluid), thus two peaks appear.

In the high-wind case (Fig. 13), the two peaks are well separated. The peak of lower frequency corresponds to the natural frequency $(7.8 \mathrm{~Hz})$ of the model. The peak of higher frequency corresponds to the frequency of the Bérnard-Kármán vortex shedding. Evidently, an increase of $\alpha$, though enhancing the sharpness of the structure frequency, does not change the Karman shedding activity at all. An increase of vibration amplitude only promotes the energy content by slightly widening the peaks.

The lock-in case (Fig. 12), by contrast, is much more complicated. At $V_{r}=11$, two frequencies are close to each other, and a clear merging of the peaks is observed. The merging, 
or the resonance, between the structure and the Bérnard-Kármán vortex shedding frequencies amplifies the peak amplitude at the reduced frequency of $\sim 0.09$. With an increased vibration amplitude, the second peak becomes increasingly merged into the first peak, while the frequency of the latter remains unchanged, hence the name lock-in. In the $\sigma_{y} / D=18 \%$ case, a third peak also appears around 0.18 , which is believed to be the second harmonic of the primary lock-in frequency. Finally, as expected, $\alpha$ has minimal effects on the lock-in behavior.

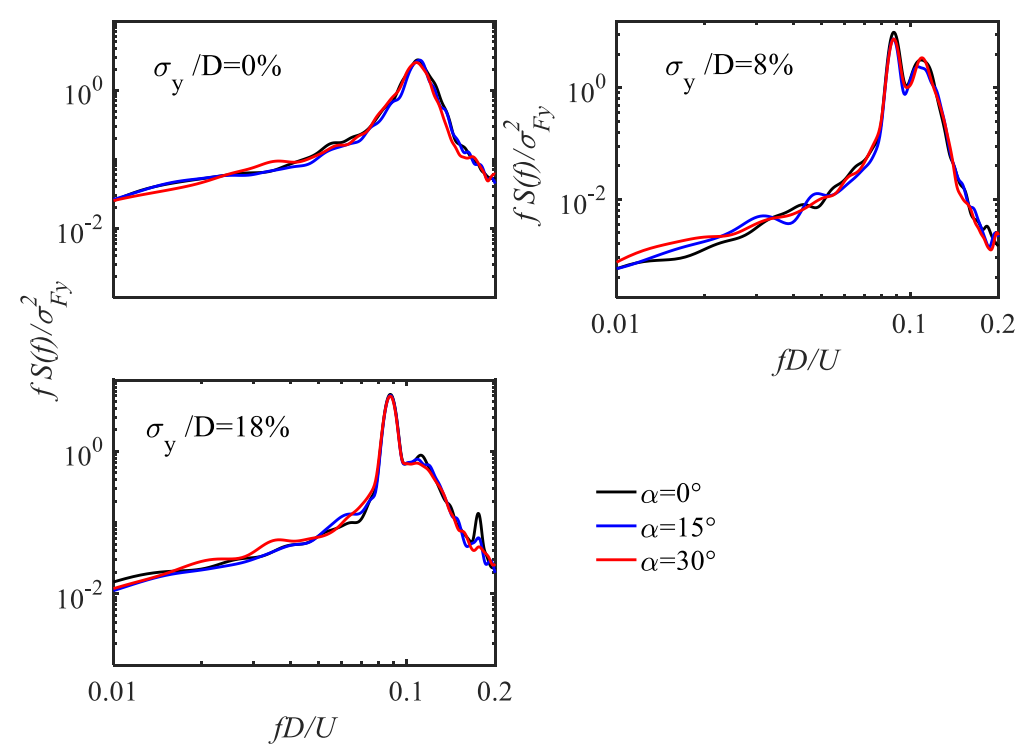

Fig. 12 Generalized force spectrum in the crosswind direction

$$
\left(V_{r}=11\right)
$$




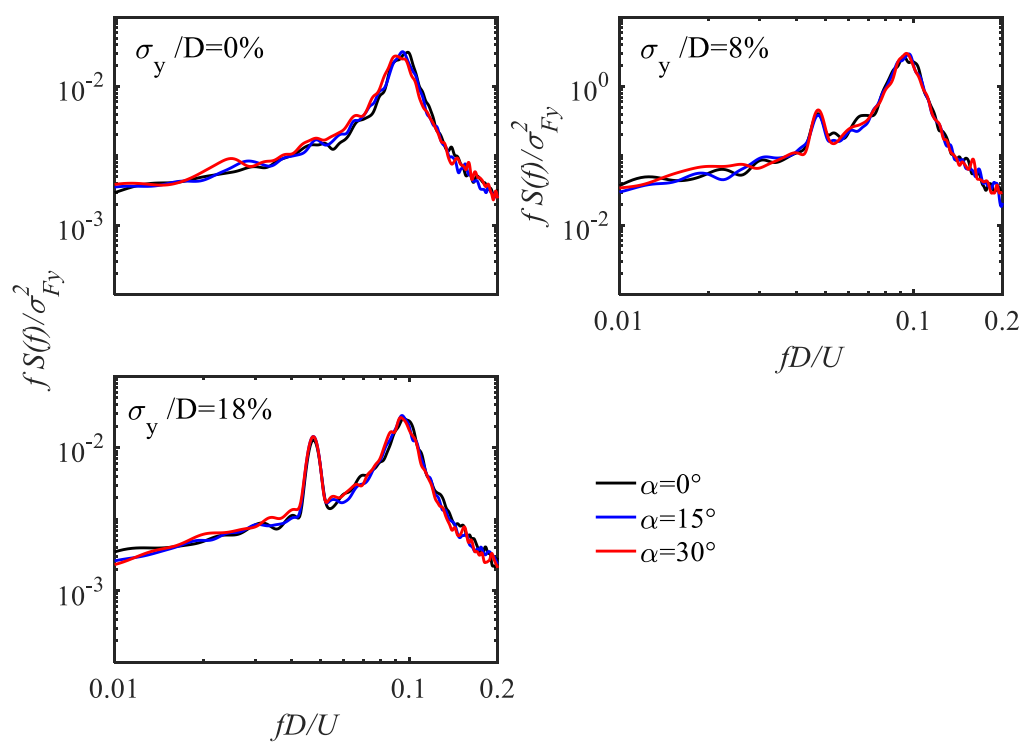

Fig. 13 Generalized force spectrum in the crosswind direction

$$
\left(V_{r}=18\right)
$$

\section{$354 \quad$ Pointwise Pressure Spectra}
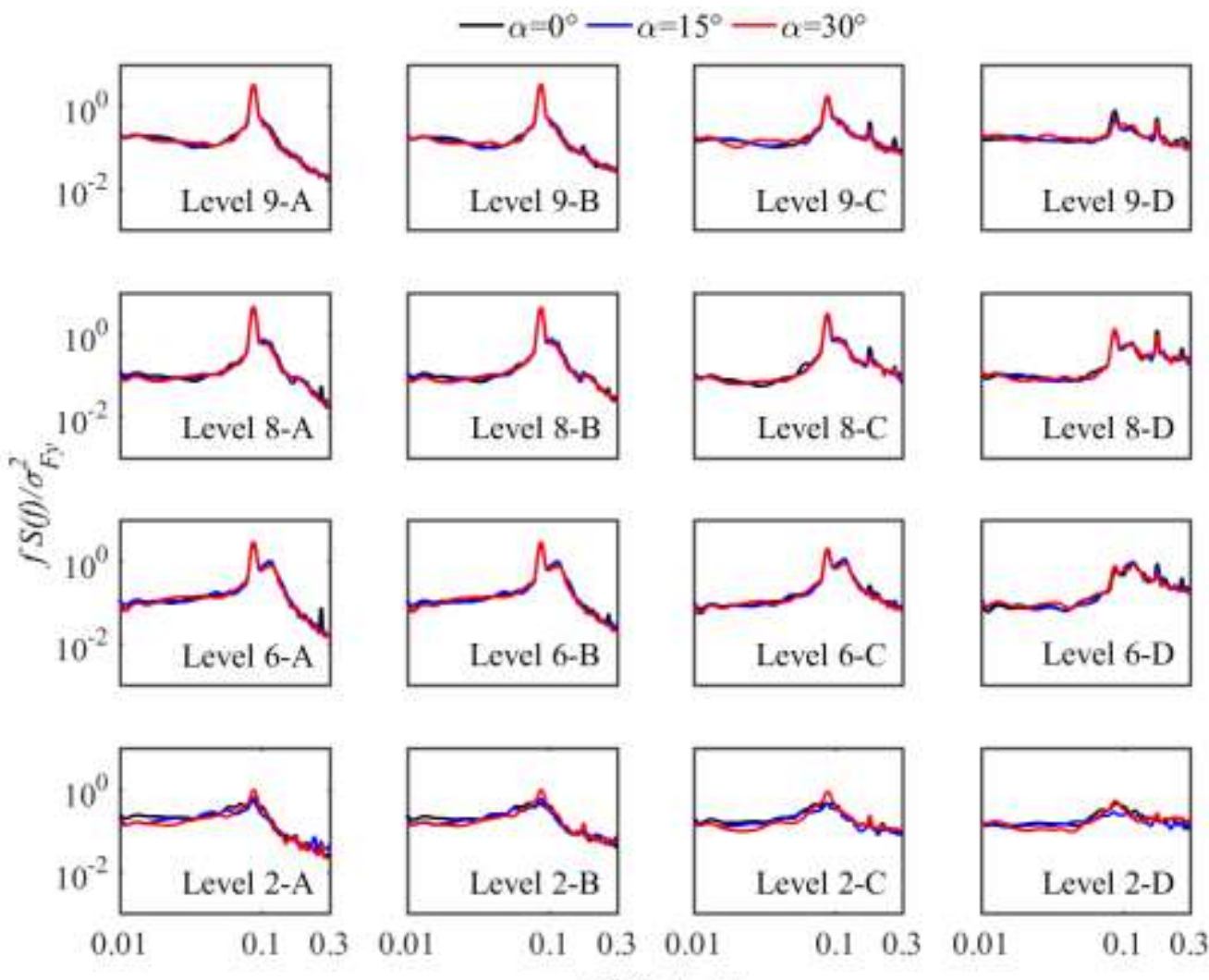

$f D / U V_{r}=11$ 

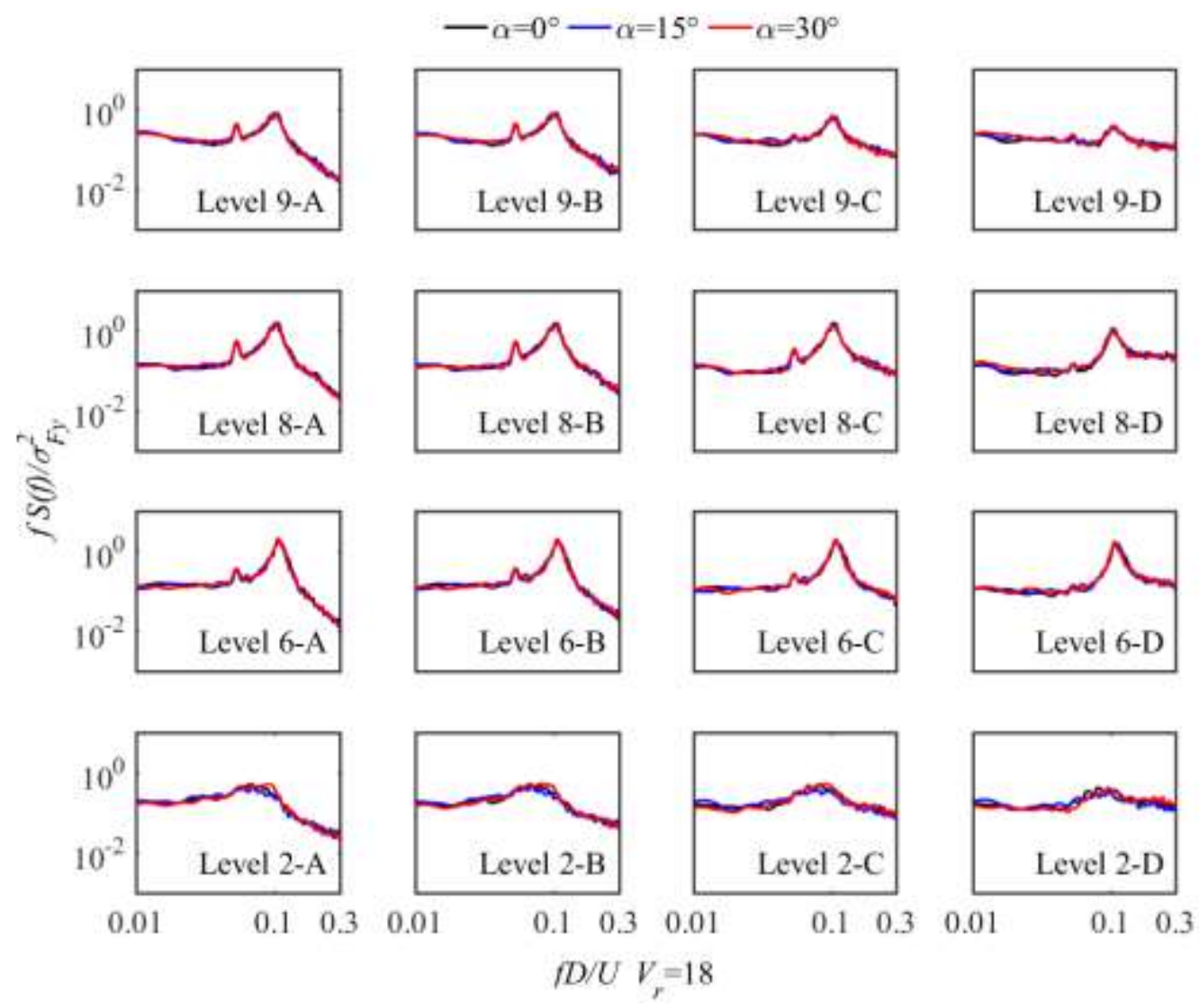

Fig. 14 Spectra of pointwise pressures on the side face of the test model at different levels: $V_{r}=11$ and $V_{r}=18$

Fig. 14 presents the pointwise pressure spectra at different altitudes. At the lock-in speed, the merged main peak becomes increasingly sharp with height, corresponding to the intensifying vortex shedding activity approaching the free-end. In addition, due to the redistribution of energy $[19,20]$, the peak values of downstream of the test model are significantly smaller than those upstream. However, at the high wind speed, the two peaks are well separated, marking the disentangled frequencies beyond the lock-in region.

Fig. 15 shows the pointwise spectra of point A of the model at different heights in the lock-in speed $\left(V_{r}=11\right)$, which is at the leading edge of the model. The Karman shedding phenomenon 
intensifies with height and is insensitive with $\alpha$ except at Level 2. Near the base, a notable proportionality of vortical activity and $\alpha$ is observed. In general, the prominent effects of transverse inclination is limited to the model base where the Karman shedding is heavily disrupted.

\subsection{Strouhal number}

Fig. 16 shows the altitudinal Strouhal Number $(S t)$ of the model at high wind speed $\left(V_{r}=18\right)$.

The variations of $S t$ with $\alpha$ are consistent with previously observed patterns on the mean pressure and RMS lift: the transverse inclination angle affect only the bottom half of the model. In the upper half, $S t$ is uniform at 0.11 , and it drops to as low as 0.07 near the base. Interestingly, all inclined cases exhibit greater St compared to the vertical case except for $\alpha=10^{\circ}$. The changing of $S t$ at the base signals a shift in the dominant vortical mechanism, which will be further reinforced by upcoming observations.

Fig. 17 presents a comparison of the Karman shedding frequency of the forced-vibration and rigid tests. In the rigid model, the frequency exhibits a linear relationship with wind speed. By contrast, in the forced-vibration test, although the linearity is the same at high wind speeds,

381 fluctuations exist at low speeds. Most notably, inside the lock-in region, the shedding frequency is locked with the natural frequency of the structure $(7.8 \mathrm{~Hz}$ ) for a range of velocities. The platform at $7.8 \mathrm{~Hz}$, after persisting for a range of velocities, is suddenly broken to meet the 
384 linearity. This vivid manifestation of the lock-in phenomenon is also evident for all $\alpha$ 's and is 385 in agreement with previous studies[14, 18, 21].

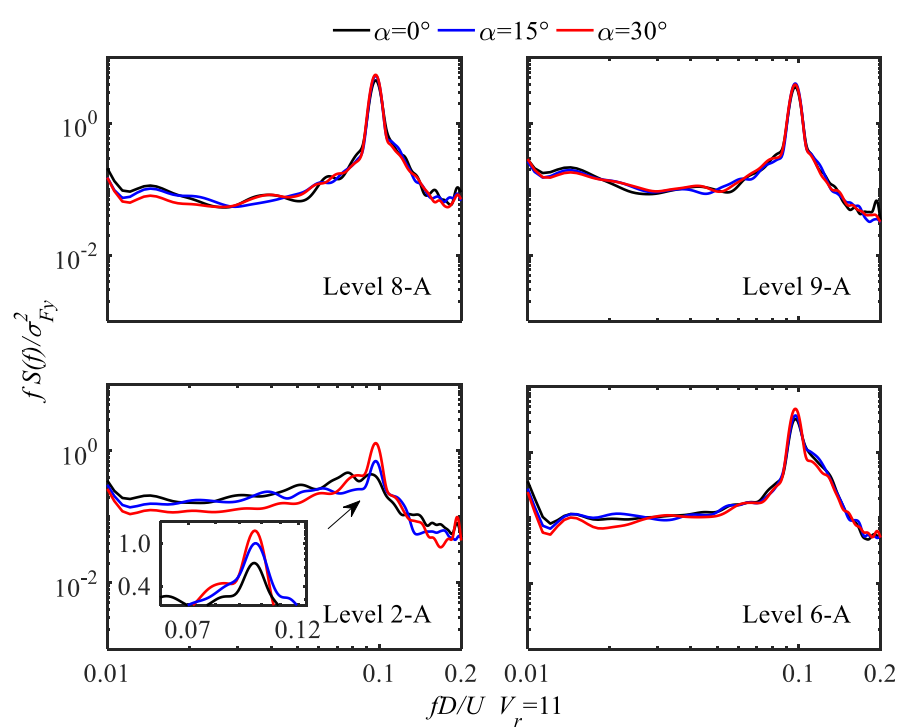

Fig. 15 Pointwise spectra analysis at points $\mathrm{A}$ at different heights

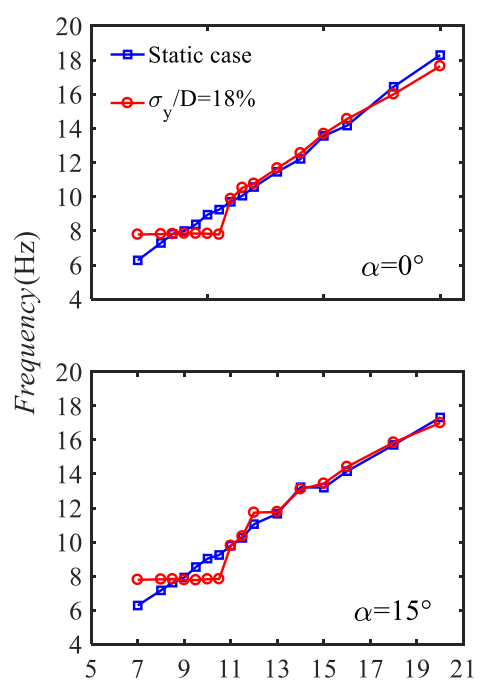

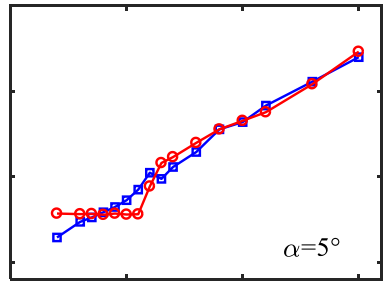

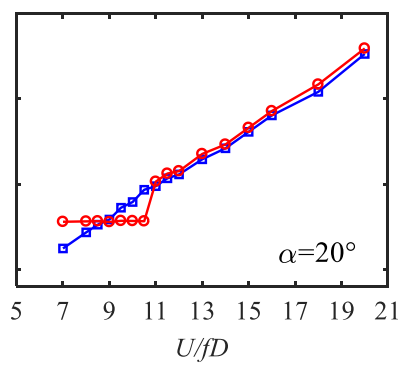

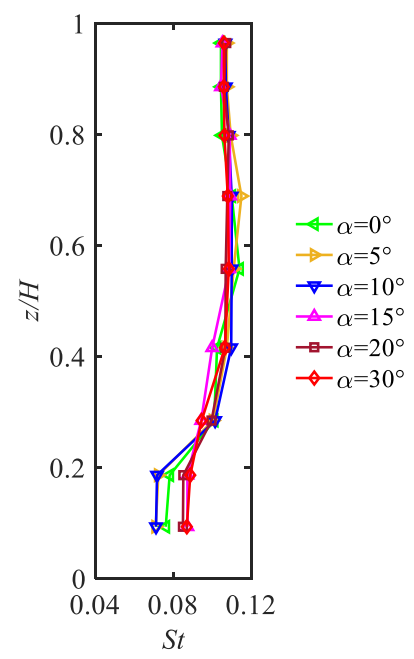

Fig. 16 Local Strouhal numbers $\left(V_{r}=18\right)$
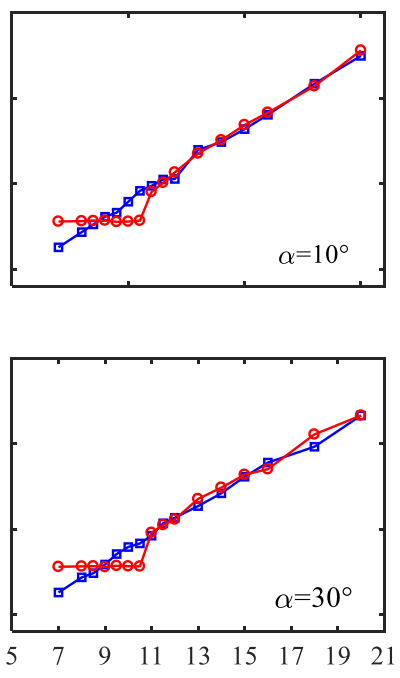

Fig. 17 Comparison of static and forced-vibration tests with vortex shedding frequency 

in crosswind direction, and the aerodynamic damping plays a key role in all of them [22-26]. Therefore, it is necessary to study the aerodynamic damping of transversely inclined structures.

392 The governing equation of motion of a prism in the crosswind direction is expressed as:

$$
m(z) \ddot{v}(z, t)+2 m(z) \xi_{v} \omega_{v} \dot{v}(z, t)+k v(z, t)=P(z, t)
$$

393 where $m(z)$ is the mass per unit height at altitude $z, \xi_{v}$ is the damping ratio, $\omega_{v}$ is the natural angular frequency, $v$ is the generalized crosswind displacement, and $k$ is the structure stiffness. $P(z, t)$ is the local crosswind force that consists of the aerodynamic and motioninduced (self-excited) components, which can be expressed as:

$$
P(z, t)=0.5 \rho U^{2}\left[C_{M}(z, t)+C_{L}(z, t)\right] D
$$

397 where $C_{M}(z, t)$ and $C_{L}(z, t)$ are the local crosswind aerodynamic force and the motioninduced force coefficients at height $z$, respectively. $\rho$ is the density of air; $U$ is the wind speed at point of reference. In the forced-vibration test, the time history tip response can be expressed as $y(t)=$ $\hat{y} \cos 2 \pi f t$, where $\hat{y}$ is the maximum standard derivation of the tip response. The motioninduced force consists of the aerodynamic stiffness term in phase with displacement and the aerodynamic damping term in phase with velocity. For super tall buildings, the aerodynamic 
405 stiffness term is usually small and can be ignored in crosswind direction. Therefore, only the

406 aerodynamic damping term is of concern [27]. Accordingly, Eq. 5 can be expressed as:

$$
P(z, t)=S_{1} \cos 2 \pi f t+S_{2} \sin 2 \pi f t
$$

407 where the aerodynamic damping force coefficient $S_{1}$ is expressed as:

$$
S_{1}=\frac{2}{T} \int_{0}^{T} P(z, t) \frac{\dot{y}}{2 \pi f \hat{y}} d t
$$

408 Since

$$
\frac{y}{\hat{y}}=\cos 2 \pi f t \quad \text { and } \quad \frac{\dot{y}}{\hat{y}}=\sin 2 \pi f t
$$

409 The local damping force coefficient $\chi(z)$ is

$$
\chi(z)=\frac{2 S_{1}}{\rho D U^{2}}
$$

410 The generalized damping force coefficient $\kappa$ is

$$
\kappa=\frac{\int_{0}^{H} \mu(z) \phi(z) d z}{H}
$$

411 The normalized damping coefficient $\chi$ is

$$
\chi=\xi_{b} / \eta=-\frac{3}{4} \frac{D}{\hat{y}}\left(\frac{U}{D w_{v}}\right)^{2} \kappa
$$

412 where $\eta=\rho D^{2} / m_{v}, \xi_{b}$ is aerodynamic damping ratio, $m_{v}$ is the unit mass. 


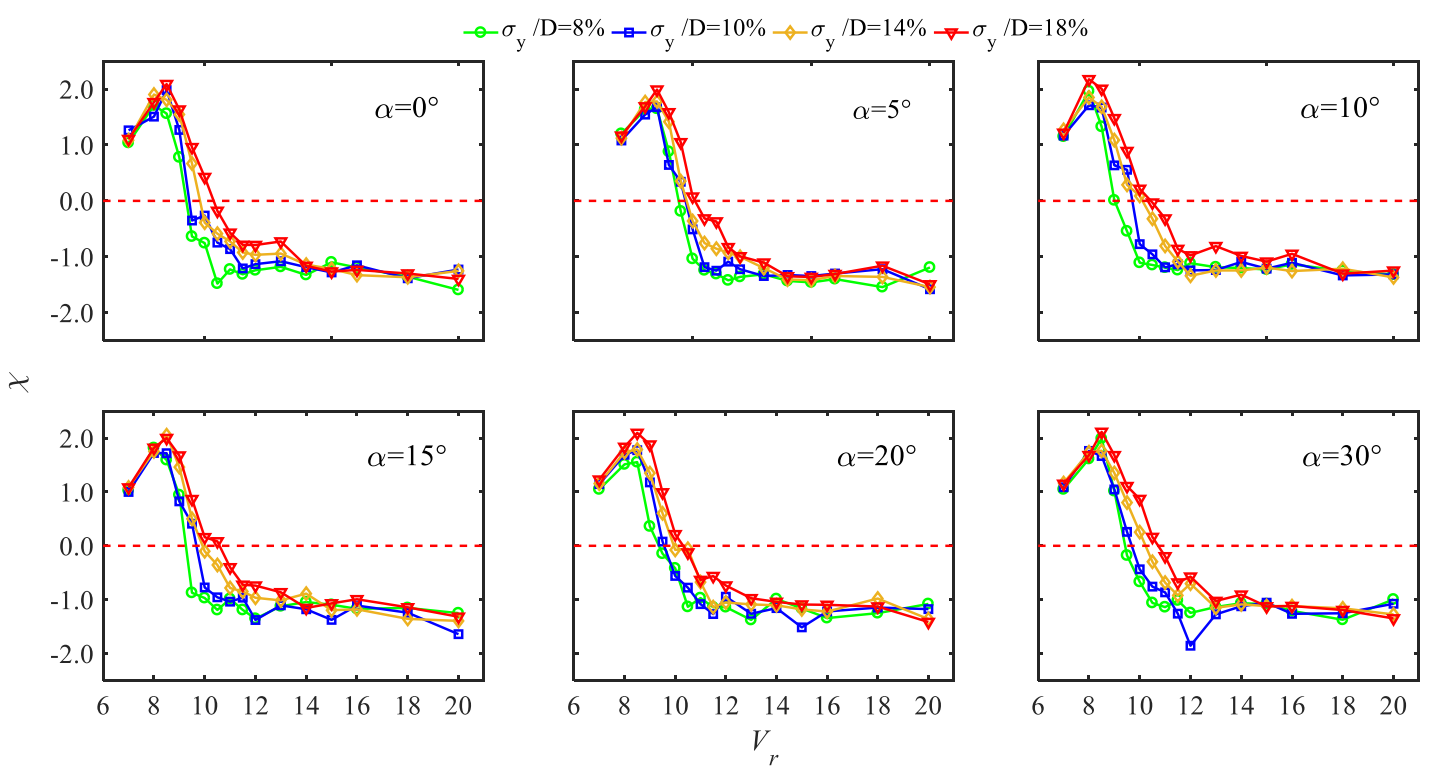

Fig. 18 Generalized aerodynamic damping for transversely inclined prisms
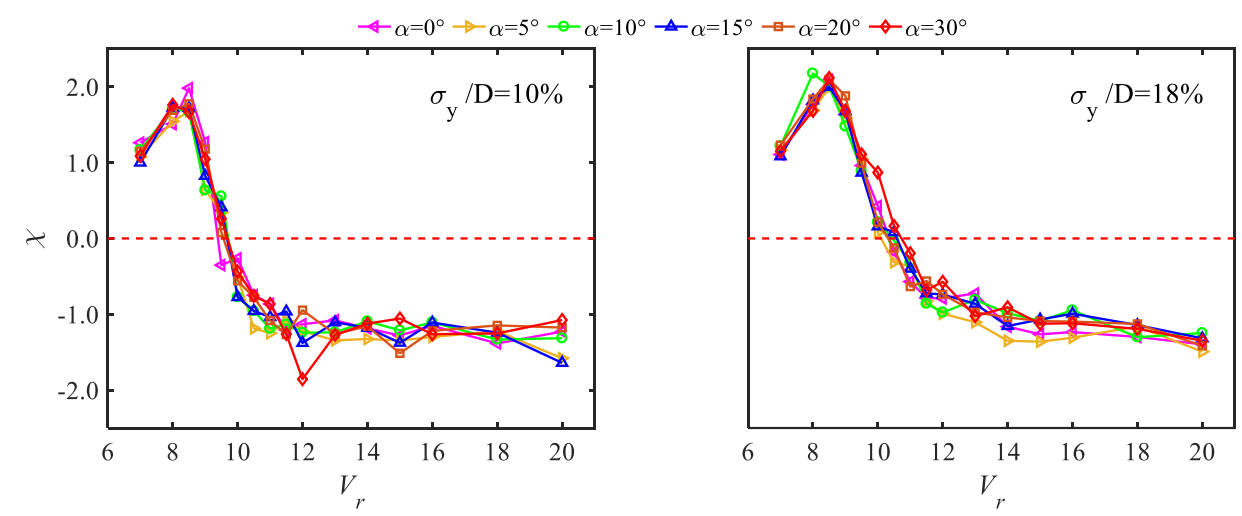

Fig. 19 Comparison of generalized aerodynamic damping at different inclination angles

415 Fig. 18 presents the generalized aerodynamic damping of the model, which is obtained from

416 Eq. (10) at different vibration amplitude, wind speeds and $\alpha$. In general, the generalized

417 aerodynamic damping $\chi$ is positive in the lock-in region, while it is negative in the high wind

418 speeds. The positive aerodynamic coefficient signifies the self-containing nature of the

419 crosswind vibration. Incidentally, for the zero-ordinate of $\chi$ marks the end of the lock-in, hence prominent vibration. Accordingly, if the aeroelastic effect is ignored (i.e., without 

aerodynamic damping), there will be over- and underestimations in the predicted response at low and high wind speeds, respectively.

Both $\alpha$ the vibration amplitude has limited influence on $\chi$, except the latter mildly shifts the corresponding velocity of the zero-ordinate to higher magnitudes. Perhaps the generalized aerodynamic damping force coefficient presented in Fig. 19 illustrates this insensitivity to a better depiction. The curves of generalized $\chi$ are closely self-similar, displaying a lucid message that $\alpha$ and vibration amplitude have minimal influence on $\chi$. In general, wind speed is primary determinant of the behaviors of $\chi$.

\subsubsection{Local aerodynamic damping coefficients}

According to Eq. (9), the local aerodynamic damping of the structure with $\sigma_{y} / D=14 \%$ is obtained in Fig. 20. At sufficiently high levels, the local $\chi$ curves resemble those of the generalized $\chi$ from Fig. 19 and Fig. 20. Nonetheless, near the model base (Levels 2 and 4), $\chi(z)$ is of much less magnitude compared to their high-level counterparts. At Level 2, on contrary to all other cases, $\chi(z)$ barely crosses the zero-ordinate. Perhaps another unexpected observation on the altitudinal effect on $\chi(z)$ is that the maximum local $\chi$ does not occur at Level 9, but rather at Levels 7 and 8. Readers are also remined by similar observations made on the local RMS lift. After consulting fluid mechanics and bluff-body aerodynamics, logical explanations are found for these unintuitive observations. 
442 The oddities of Level 2 and Level 9 are attributed to different mechanisms either dominating 443 or affecting the aerodynamic behaviors of the structure. As is well-known, the predominant 444 driving mechanism of this configuration, including the excitation of the lock-in phenomenon, 445 is the Bérnard-Kármán vortex shedding. The self-similar local $\chi(z)$ curves, as well as all 446 previous observations, support this notion. The first exception is at the model base. Near the 447 model base, the Bérnard-Kármán vortex shedding is completely overshadowed by fix-end 448 vortical activities like the horse-shoe vortex shedding. As the result, the entire array of 449 aerodynamic parameters at the model base, including the aforementioned mean pressure 450 distribution, RMS lift coefficient, Strouhal number, and aerodynamic damping are 451 fundamentally different from higher levels. As for the other end, the free-end of the model, the 452 Bérnard-Kármán vortex shedding is heavily affected by three-dimensional effects like the downwash axial flow. However, the degree of the influence is not as overwhelming as the horseshoe vortex. Consequently, as reflected by the local RMS lift, local Strouhal number, and here the local aerodynamic coefficient, the Karman shedding maintains its dominance but with a compromised intensity. The maximum local RMS lift and local $\chi$ are also recorded slight below the free-end. Nonetheless, and in spite the different driving mechanisms near both ends, the overall lock-in phenomenon dictates the intensity of all aerodynamic activities, thus wind speed becomes the most decisive factor.

461 Perhaps this is also a good point to elucidate the aerodynamic effect of the transverse 462 inclination. As all evidence suggest, the transverse inclination induces a bi-polar step-change to the aerodynamic behaviors of the prism. A prescription of any degrees of $\alpha$ projects a notable 
464 impact to the bottom half of the model, while the upper half remains unaffected. The impact 465 consists of drastically enhanced base RMS lift, elevated base Strouhal number, and here the 466 extended range of positive $\chi(z)$. All of these suggest a universal tenet: the transverse 467 inclination intensifies fix-end aerodynamic phenomena like the horseshoe vortex, causing a more erratic wind field near, and stronger loadings onto, the structure base. Yet, once the initial perturbation, the degree of the intensification is influenced by neither the angle of inclination nor the amplitude of tip vibration. The practical implications are that structural inclinations of any orientation, as it easily changes with the wind attack angle, may cause unpredictable wind environment at the pedestrian level, and demand more safety reinforcement near the build base. inclined structures.

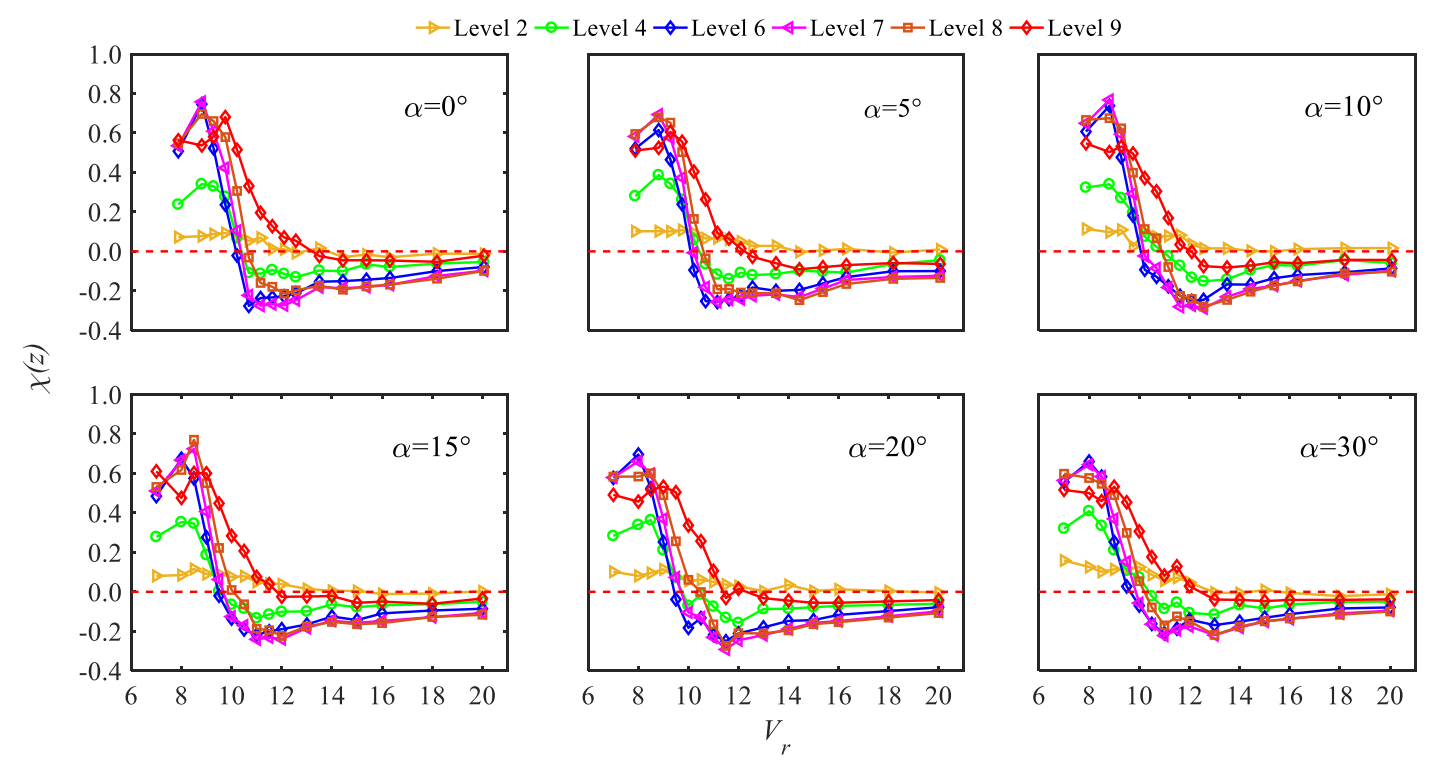

Fig. 20 Local aerodynamic damping for transversely inclined structures 
In this final section, based on the unsteady aerodynamic force obtained from the forced-

479 vibration test, the response of the structure is predicted according to the frequency domain 480 method [12].

$$
\begin{gathered}
S_{u}(w)=S_{F u}(w) \cdot H^{2}(w) \\
H^{2}(w)=\left\{\left(1-\left(f_{u} / f_{s}\right)^{2}\right)^{2}+4\left(\xi_{b}+\xi_{0}\right)^{2}\left(f_{u} / f_{s}\right)^{2}\right\}^{-1}
\end{gathered}
$$

481 where $S_{u}(w)$ is the displacement spectrum, $S_{F u}(w)$ is the force spectrum, $H^{2}(w)$ is the 482 mechanical admittance, $\xi_{b}$ is the aerodynamic damping ratio identified by Eq. (11), $\xi_{0}$ is the structural damping ratio, and $f_{s}$ is the natural frequency $(7.8 \mathrm{~Hz})$. (14):

$$
\sigma_{y}=\left\{\frac{1}{K_{s}} \int_{0}^{\infty} S_{u}(w) d w\right\}^{1 / 2}
$$

487 where $K_{s}$ is the model stiffness and $\sigma_{y}$ is the tip response. 


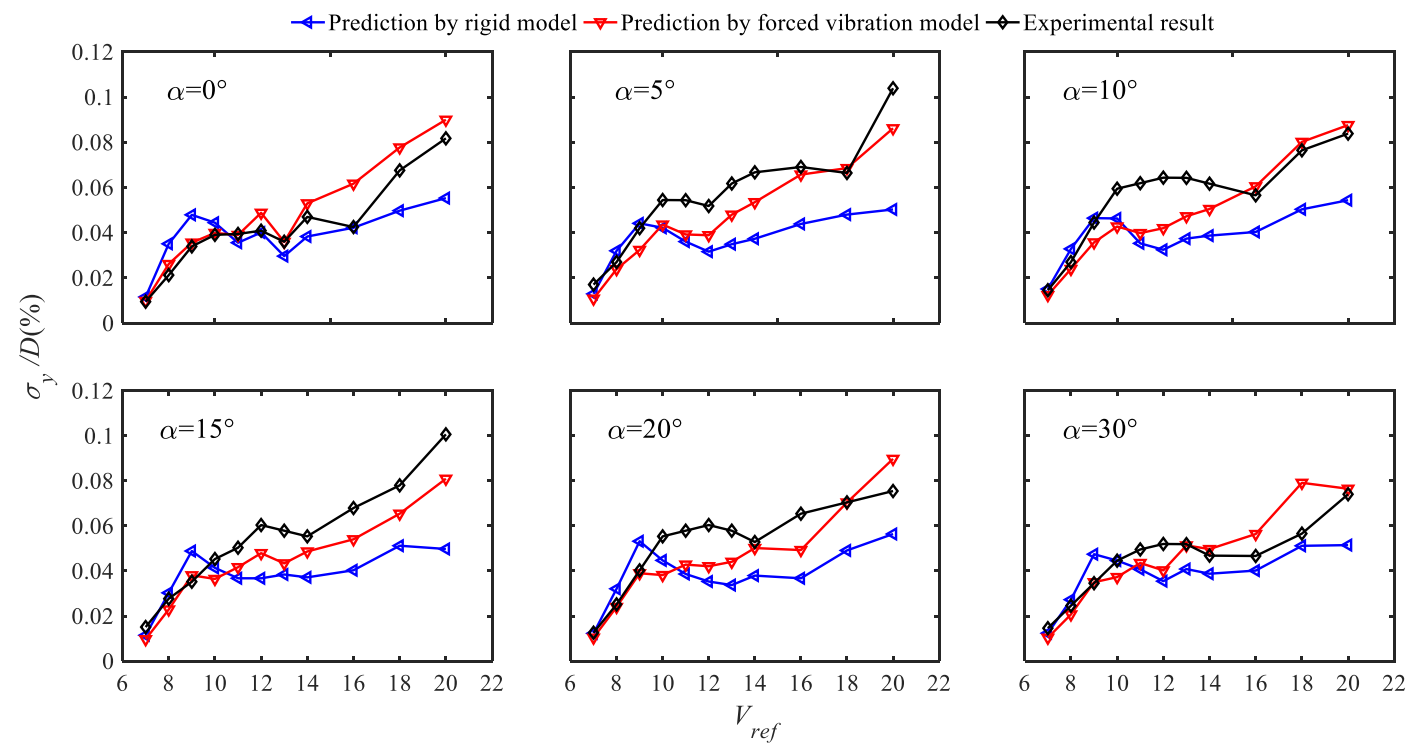

Fig. 21 Comparison between the predicted standard deviation of the test model tip response and the measured values of the aeroelastic test

Fig. 21 shows the comparison between the predicted and measured response at different $\alpha$ 's. As expected, the predicted responses by forced-vibration and rigid tests increase with wind speed, generally matching the trend of the experimental result. Nevertheless, notable differences can be found in the predicted and experimental responses. For the vertical case, both predictions closely match the measured response, except the forced-vibration response is almost exact in the lock-in region and overestimates at high wind speeds. Compared to the underestimating rigid test, the forced-vibration provides more adequate predictions for structural safety. With inclination, the forced-vibration prediction generally outperforms the rigid prediction by tallying smaller differences with the experimental value. In specific cases, such as at $V_{r}=20$ and $\alpha=20^{\circ}$ and $30^{\circ}$, the forced-vibration prediction outperforms the rigid one by as much as $28.9 \%$ and $27.2 \%$. This is because the rigid prediction completely ignores the fluid-structure interaction. On this note, even the forced-vibration prediction universally underestimates the actual response, especially in the lock-in region. The primary cause is that 
501 the forced-vibration experiment considers only the one-way fluid-structure interaction by ignoring the flow-induced motion. To provide better predictions, future effort must rely on the

503 results from a free-vibration, fully aeroelastic test.

\section{Conclusion}

506 In this work, unsteady aerodynamic characteristic of a transversely inclined square prisms were investigated using the forced-vibration technique, in which different wind speeds, inclination angles, and oscillation amplitudes have been tested. Specifically, the configuration's aerodynamic loading, vortical activities, and aerodynamic damping have been analysis. Subsequently, the predicted responses of the forced-vibration and rigid tests were compared with empirical values.

513 The central finding of this work is the discovery of the Base Intensification phenomenon. It refers to the intensification of the aerodynamic loading, vortical activities, and aerodynamic

515 damping of the lower half of a structure when subjected to transverse inclination. The 516 phenomenon is incurred by any prescription of transverse inclination at any degree. Yet,

517 interestingly, once incurred, it does not intensify nor deteriorate with the angle of inclination and tip vibration amplitude. This bi-polar step-change is associated with the promotion of fixend effects like the horseshoe vortex shedding. The upper half of the structure, dominated by the Bérnard-Kármán vortex shedding, is generally unaffected by transverse inclination. 
522 Another major finding is that the wind speed is the most dominant mechanism of the 523 aerodynamic behaviors of the configuration. In the lock-in region, the crosswind vibrations,

524 including the Base Intensification phenomenon, are significantly amplified. With a continuous

525 increase of wind speed, the configuration gradually resorts to the quasi-steady linear behavior.

527 Based on empirical results, the response of the inclined model is predicted by the force528 vibration and rigid test. The force-vibration offers a great improvement to the prediction based 529 on the rigid test. However, since the former only considers the one-way fluid-structure 530 interaction, the prediction still tends to underestimate actual response, particularly inside the 531 lock-in region.

533 Finally, some engineering implications and future forecasts deserve a brief discussion. Due to the Base Intensification phenomenon, the safety of inclined structures, regardless of inclination

535 orientation, demands more engineering caution. Potential perils like erratic pedestrian-level 536 winds and excessive aerodynamic load on structure base may take place given changes in the 537 wind attack angle. Moreover, future effort shall improve the response prediction by resorting to the free-vibration tests, such that the safety of inclined structures may be better guaranteed 539 in the design stage. 
542 The work described in this paper was supported by the National Natural Science Foundation 543 of China (Grant No.: 51908090), the Fundamental Research Funds for the Central Universities 544 (Project No.: 2019CDXYTM0032), the Natural Science Foundation of Chongqing, China 545 (Grant No.: cstc2019jcyj-msxm0639, cstc2020jcyj-msxmX0921), the Key project of 546 Technological Innovation and Application Development in Chongqing (Grant No.: 547 cstc2019jscx-gksb0188). The authors appreciate the use of the testing facility, as well as the 548 technical assistance provided by the CLP Power Wind/Wave Tunnel Facility at the Hong Kong 549 University of Science and Technology. The authors would also like to express our sincere 550 thanks to the Design and Manufacturing Services Facility (Electrical and Mechanical 551 Fabrication Unit) of the Hong Kong University of Science and Technology for their help in 552 manufacturing the test rig of the forced vibration wind tunnel test system. 


\section{Funding}

The work described in this paper was supported by the National Natural Science Foundation of China (Grant No.: 51908090), the Fundamental Research Funds for the Central Universities

561 (Project No.: 2019CDXYTM0032), the Natural Science Foundation of Chongqing, China (Grant No.: cstc2019jcyj-msxm0639, cstc2020jcyj-msxmX0921), the Key project of Technological Innovation and Application Development in Chongqing (Grant No.: cstc2019jscx-gksb0188).

\section{Conflict of Interest}

567 The authors declare that they have no conflict of interest.

The datasets generated during and/or analyzed during the current work are restricted by request. 
575 The custom code used during and/or analyzed during the current work are restricted by 576 provisions of the funding source.

579 All authors contributed to the study conception and design. Material preparation, data 580 collection and analysis were performed by Zengshun Chen, Jie Bai and Yemeng Xu, Jianmin 581 Hua, and Xuanyi Xue. The first draft of the manuscript was written by Cruz Y. Li and Jie Bai 582 and all authors commented on previous versions of the manuscript. All authors read and approved the final manuscript.

\section{Compliance with Ethical Standards}

586 All procedures performed in this work were in accordance with the ethical standards of the 587 institutional and/or national research committee and with the 1964 Helsinki declaration and 588 its later amendments or comparable ethical standards.

\section{Consent to Participate}

591 Informed consent was obtained from all individual participants included in the study.

\section{Consent for Publication}

594 Publication consent was obtained from all individual participants included in the study. 
596

1. Tanaka, H., Tamura, Y., Ohtake, K., Nakai, M., Chul Kim, Y.: Experimental investigation of aerodynamic forces and wind pressures acting on tall buildings with various unconventional configurations. J. Wind Eng. Ind. Aerodyn. 107-108, 179-191 (2012). https://doi.org/10.1016/j.jweia.2012.04.014

2. Hui, Y., Yuan, K., Chen, Z., Yang, Q.: Characteristics of aerodynamic forces on highrise buildings with various façade appurtenances. J. Wind Eng. Ind. Aerodyn. 191, 76-90 (2019). https://doi.org/10.1016/j.jweia.2019.06.002

3. Carassale, L., Freda, A., Marrè-Brunenghi, M.: Experimental investigation on the aerodynamic behavior of square cylinders with rounded corners. J. Fluids Struct. 44, 195-204 (2014). https://doi.org/10.1016/j.jfluidstructs.2013.10.010

4. Hu, Gang, Tse, K.T., Kwok, K.C.S., Chen, Z.S.: Pressure measurements on inclined square prisms. Wind Struct. 21, 383-405 (2015).

https://doi.org/10.12989/WAS.2015.21.4.383

5. Hu, G., Tse, K.T., Kwok, K.C.S.: Galloping of forward and backward inclined slender square cylinders. J. Wind Eng. Ind. Aerodyn. 142, 232-245 (2015).

https://doi.org/10.1016/j.jweia.2015.04.010

6. Mannini, C., Marra, A.M., Bartoli, G.: Experimental investigation on VIV-galloping interaction of a rectangular 3:2 cylinder. Meccanica. 50, 841-853 (2015). https://doi.org/10.1007/s11012-014-0025-8

7. Chen, Z., Fu, X., Xu, Y., Li, C.Y., Kim, B., Tse, K.T.: A perspective on the aerodynamics and aeroelasticity of tapering: Partial reattachment. J. Wind Eng. Ind. Aerodyn. 212, 104590 (2021). https://doi.org/10.1016/j.jweia.2021.104590

8. Cooper, K.R., Nakayama, M., Sasaki, Y., Fediw, A.A., Resende-Ide, S., Zan, S.J.: Unsteady aerodynamic force measurements on a super-tall building with a tapered cross section. J. Wind Eng. Ind. Aerodyn. 72, 199-212 (1997). https://doi.org/10.1016/S01676105(97)00258-4

9. Chen, Z., Tse, K.T., Kwok, K.C.S., Kareem, A.: Aerodynamic damping of inclined slender prisms. J. Wind Eng. Ind. Aerodyn. 177, 79-91 (2018). https://doi.org/10.1016/j.jweia.2018.04.016

10. Zou, L., Li, F., Song, J., Shi, T., Liang, S., Mercan, O.: Investigation of torsional aeroelastic effects on high-rise buildings using forced vibration wind tunnel tests. J. Wind Eng. Ind. Aerodyn. 200, 104158 (2020). https://doi.org/10.1016/j.jweia.2020.104158

11. Kim, Y.C., Lo, Y.L., Chang, C.H.: Characteristics of unsteady pressures on slender tall building. J. Wind Eng. Ind. Aerodyn. 174, 344-357 (2018).

https://doi.org/10.1016/j.jweia.2018.01.027 
631 12. Chen, Z., Huang, H., Tse, K.T., Xu, Y., Li, C.Y.: Characteristics of unsteady

632 aerodynamic forces on an aeroelastic prism: A comparative study. J. Wind Eng. Ind.

633 Aerodyn. 205, 104325 (2020). https://doi.org/10.1016/j.jweia.2020.104325

634 13. Chen, Z., Tse, K.T., Kwok, K.C.S., Kareem, A., Kim, B.: Measurement of unsteady 635 aerodynamic force on a galloping prism in a turbulent flow: A hybrid aeroelastic-pressure

636 balance. J. Fluids Struct. 102, 103232 (2021).

637 https://doi.org/10.1016/j.jfluidstructs.2021.103232

638 14. Chen, Z., Tse, K.T., Kwok, K.C.S.: Unsteady pressure measurements on an 639 oscillating slender prism using a forced vibration technique. J. Wind Eng. Ind. Aerodyn. 170, 640 81-93 (2017). https://doi.org/10.1016/j.jweia.2017.08.004

$641 \quad$ 15. Chen, Z., Tse, K.T., Hu, G., Kwok, K.C.S.: Experimental and theoretical 642 investigation of galloping of transversely inclined slender prisms. Nonlinear Dyn. 91, 10236431040 (2018). https://doi.org/10.1007/s11071-017-3926-y

644 16. Holmes, J.D.: Wind Loading of Structures. CRC Press (2018)

645 17. Lin, N., Letchford, C., Tamura, Y., Liang, B., Nakamura, O.: Characteristics of wind 646 forces acting on tall buildings. J. Wind Eng. Ind. Aerodyn. 93, 217-242 (2005).

647 https://doi.org/10.1016/j.jweia.2004.12.001

648 18. Bearman, P.W., Obasaju, E.D.: An experimental study of pressure fluctuations on 649 fixed and oscillating square-section cylinders. J. Fluid Mech. 119, 297-321 (1982).

650 https://doi.org/10.1017/S0022112082001360

651 19. Kareem, A.: Measurements of pressure and force fields on building models in

652 simulated atmospheric flows. Sixth US Natl. Conf. Wind Eng. 36, 589-599 (1990).

653 https://doi.org/10.1016/0167-6105(90)90341-9

654 20. Kareem, A., Cermak, J.E.: Pressure fluctuations on a square building model in 655 boundary-layer flows. J. Wind Eng. Ind. Aerodyn. 16, 17-41 (1984).

656 https://doi.org/10.1016/0167-6105(84)90047-3

657 21. Chen, Z., Huang, H., Xu, Y., Tse, K.T., Kim, B., Wang, Y.: Unsteady aerodynamics 658 on a tapered prism under forced excitation. Eng. Struct. 240, 112387 (2021).

659 https://doi.org/10.1016/j.engstruct.2021.112387

660 22. Steckley, A., Vickery, B.J., Isyumov, N.: On the measurement of motion induced 661 forces on models in turbulent shear flow. J. Wind Eng. Ind. Aerodyn. 36, 339-350 (1990). 662 https://doi.org/10.1016/0167-6105(90)90318-7

663 23. Vickery, B.J., Steckley, A.: Aerodynamic damping and vortex excitation on an 664 oscillating prism in turbulent shear flow. J. Wind Eng. Ind. Aerodyn. 49, 121-140 (1993).

665 https://doi.org/10.1016/0167-6105(93)90009-D 
666 24. Dai, H.L., Abdelmoula, H., Abdelkefi, A., Wang, L.: Towards control of cross-flow667 induced vibrations based on energy harvesting. Nonlinear Dyn. 88, 2329-2346 (2017).

668 https://doi.org/10.1007/s11071-017-3380-x

669 25. Abdel-Rohman, M.: Effect of Unsteady Wind Flow on Galloping of Tall Prismatic 670 Structures. 22

671 26. Dai, H.L., Abdelkefi, A., Wang, L., Liu, W.B.: Control of cross-flow-induced 672 vibrations of square cylinders using linear and nonlinear delayed feedbacks. Nonlinear Dyn. 673 78, 907-919 (2014). https://doi.org/10.1007/s11071-014-1485-z

674 27. Chen, X.: Estimation of stochastic crosswind response of wind-excited tall buildings 675 with nonlinear aerodynamic damping. Eng. Struct. 56, 766-778 (2013).

676 https://doi.org/10.1016/j.engstruct.2013.05.044

677

678 